\title{
Extended calculations of energy levels, radiative properties, and lifetimes for oxygen-like Mo XXXV
}

\author{
Kai Wang, ${ }^{\mathrm{a}, \mathrm{b}}$, Wei Zheng ${ }^{\mathrm{a}}$, Xiao Hui Zhao*,a Zhan Bin Chen ${ }^{\dagger, \mathrm{c}}$, Chong Yang Chen ${ }^{\mathrm{b}}$, Jun Yan ${ }^{\mathrm{d}}$ \\ ${ }^{a}$ Hebei Key Lab of Optic-electronic Information and Materials, The College of Physics Science and Technology, Hebei University, \\ Baoding 071002, China \\ ${ }^{b}$ Shanghai EBIT Lab, Key Laboratory of Nuclear Physics and Ion-beam Application, Institute of Modern Physics, Department of \\ Nuclear Science and Technology, Fudan University, Shanghai 200433, China \\ ${ }^{c}$ School of Science, Hunan University of Technology, Zhuzhou, 412007, China \\ ${ }^{d}$ Institute of Applied Physics and Computational Mathematics, Beijing 100088, China
}

\section{'Abstract}

Employing two state-of-the-art methods, second-order many-body perturbation theory and multiconfiguration Dirac-Fock, highly accurate calculations are performed for the lowest 318 fine-structure levels arising from the $2 s^{2} 2 p^{4}, 2 s 2 p^{5}, 2 p^{6}, 2 s^{2} 2 p^{3} 3 l, 2 s 2 p^{4} 3 l, 2 p^{5} 3 l$, and $2 s^{2} 2 p^{3} 4 l$ configurations in O-like Mo XXXV. Complete and consistent atomic data, including excitation energies, lifetimes, wavelengths, and E1, E2, M1 line strengths, oscillator strengths, and transition rates among these 318 levels are provided. Comparisons are made between the present two data sets, as well as with other available experimental and theoretical values. The present data are accurate enough for identification and deblending of emission lines involving the $n=3,4$ levels and are also useful for modeling and diagnosing fusion plasmas. These data can be considered as a benchmark for other calculations.

Key words: atomic data; O-like Mo XXXV; many-body perturbation theory; multiconfiguration Dirac-Fock method

*xhzhao@hbu.edu.cn

†chenzb008@qq.com 


\section{Introduction}

Walls of fusion reactors often contain alloys of molybdenum, and ions of Mo are present in the plasmas due to sputtering from the walls [1, 2]. Therefore, in order to simulate and diagnose plasmas that contain Mo as a constituent, accurate atomic data for different Mo ions are required. In view of this, soft X-ray emission lines from molybdenum plasmas generated by dual laser pulses were measured for different Mo ions [3]. Feldman et al. [4] identified 13 lines of the $\left(1 s^{2}\right) 2 s^{2} 2 p^{4}-2 s 2 p^{5}$ transitions in a laserproduced plasma.

On the theoretical side, excitation energies and radiative transition data for the low-lying states of the $2 s^{2} 2 p^{4}, 2 s 2 p^{5}$, and $2 p^{6}$ configurations were provided by different calculations [5-9]. Atomic parameters of the $n>2$ levels are also needed for applications in plasma physics [10, 11].

The present study is to provide a complete accurate data set of energy levels, radiative transition data, and lifetimes involving high-lying levels with principal quantum numbers $n=3,4$ in O-like Mo XXXV. This study is an extension of our previous work on O-like ions [12, 13]. Excitation energies, wavelengths, line strengths, oscillator strengths, transition rates, and lifetimes are provided here for the lowest 318 levels of the $2 s^{2} 2 p^{4}, 2 s 2 p^{5}, 2 p^{6}$, $2 s^{2} 2 p^{3} 3 l(l=s, p, d), 2 s 2 p^{4} 3 l(l=s, p, d), 2 p^{5} 3 l$ $(l=s, p, d)$, and $2 s^{2} 2 p^{3} 4 l(l=s, p, d, f)$ configurations using the many-body perturbation theory (MBPT) method [9, 14 17]. In order to assess the accuracy of our MBPT calculations, the multiconfiguration Dirac-Fock (MCDF) and relativistic configuration interaction (RCI) method [18, 19] is used to calculate the corresponding data. The present study significantly increases the amount of accurate data for the $n=3,4$ levels of O-like Mo. The accuracy of our results is sufficient to directly aid and confirm experimental identifications.

\section{Calculations}

The MBPT method integrated in the FAC code [20] and the MCDF method implemented in the GRASP2K code [21,22] are used to perform the calculations. Both of the methods have been successfully used to calculate atomic parameters for L- and
M-shells systems with high accuracy [12, 13, 2344]. We only give an outline of the MBPT and MCDF calculations, since these two methods are described in our earlier work in detail.

\section{1. $M B P T$}

In the MBPT method, the Hilbert space of the system is divided into two subspaces, including a model space $M$ and an orthogonal space $N$. By means of solving the eigenvalue problem of a non-Hermitian effective Hamiltonian in the space $M$, we can get the true eigenvalues of the Dirac-Coulomb-Breit Hamiltonian. The configuration interaction effects in the $M$ space is exactly considered, and the interaction of the spaces $M$ and $N$ is accounted for with the manybody perturbation theory up to the second order. In the calculations, we include all states of the $2 s^{2} 2 p^{4}$, $2 s 2 p^{5}, 2 p^{6}, 2 s^{2} 2 p^{3} 3 l(l=s, p, d), 2 s 2 p^{4} 3 l(l=$ $s, p, d), 2 p^{5} 3 l(l=s, p, d), 2 s^{2} 2 p^{3} 4 l(l=s, p, d, f)$, and $2 s 2 p^{4} 4 s$ configurations in the model space $M$, Through the single and double (SD) virtual excitations of the states spanning the $M$ space, all interacting virtual states necessary to properly describe interelectron correlations are contained in the space $N$. The maximum $n$ values for the single/double excitations are 200/65, respectively, while the maximum $l$ value is 20 . The leading quantum electrodynamic (QED) effects are also considered in our work.

\section{2. $M C D F$}

The MCDF method has been described by Froese Fischer et al. [19]. Based on the active space (AS) approach [45] for the generation of the configuration state function (CSF) expansions, separate MCDF calculations are done for the even and odd parity states. We start the calculation without any excitation from the reference configurations which is usually referred to as the Dirac-Fock (DF) calculation. The reference configurations are the same as those included in the $M$ space of the MBPT calculations described above. Subsequently, the CSFs expansions are obtained through the SD excitations from the shells of the reference configurations up to a $n_{\max } l_{\text {max }}$ orbital, with $n_{\max } \leq 8$ and $l_{\max } \leq 5$. To reduce the number of CSFs, the $1 s^{2}$ core is closed during the relativistic self-consistent field (RSCF) calculations, but is opened during the RCI calculations, where the 
Breit interaction and QED effects, i.e., vacuum polarization and self- energy, are included in the Hamiltonian. The number of CSFs in the final even and odd state expansions are approximately 5060000 and 3930000 , respectively, distributed over the different $J$ symmetries.

To provide the $L S J$ labeling system used in databases such as the Atomic Spectra Database (ASD) of the National Institute of Standards and Technology (NIST) [46], the wave functions in both the MCDF and MBPT calculations are transformed from a $j j$-coupled $C S F$ basis into a $L S J$-coupled CSF basis using the methods developed by Gaigalas [47, 48].

\section{Results and Discussions}

The computed excitation energies for the lowest 318 levels of the $2 s^{2} 2 p^{4}, 2 s 2 p^{5}, 2 p^{6}, 2 s^{2} 2 p^{3} 3 l(l=$ $s, p, d), 2 s 2 p^{4} 3 l(l=s, p, d), 2 p^{5} 3 l(l=s, p, d)$, and $2 s^{2} 2 p^{3} 4 l(l=s, p, d, f)$ configurations from our MBPT and MCDF calculations are listed in Table 1, along with the radiative lifetimes estimated from E1, E2, and M1 transition rates, and the $L S J$ coupled and $j j$-coupled labels obtained from our calculations. Table 2 lists wavelengths $\lambda_{i j}$, and E1, E2, M1 line strengths $S_{j i}$, oscillator strengths $g_{i} f_{j i}$, and radiative rates $A_{j i}$ among the 318 energy levels along with branching fractions $\left(\mathrm{BF}_{j i}=\frac{A_{j i}}{\sum_{k=1}^{j-1} A_{j k}}\right)$, obtained from both the MBPT and MCDF methods. All the E1 and E2 values are computed in the Babushkin gauge (equivalent to the non-relativistic length form), which is considered to be more accurate than the Coulomb gauge (equivalent to the nonrelativistic velocity form).

\subsection{Excitation energies}

Since excitation energies for O-like Mo are only available for the $n=2$ levels in the previous experimental and theoretical studies, the MBPT and MCDF excitation energies are compared with the experimental values from the NIST ASD, and the other theoretical results calculated by $\mathrm{Gu}$ [7] and Vilkas et al. [9] in Table 3, The average difference with the standard deviation from the NIST values are $-249 \pm 748 \mathrm{~cm}^{-1}$ for MBPT, $-68 \pm 471 \mathrm{~cm}^{-1}$ for
MCDF, $1053 \pm 707 \mathrm{~cm}^{-1}$ for $\mathrm{Gu}$, and $1955 \pm 1758$ $\mathrm{cm}^{-1}$ for Vilkas et al., respectively. Comparing with the previous calculations, there is generally a better agreement between the NIST values and our MBPT (MCDF) results due to the larger extent of the electron correlation effects included in our work.

For the remaining levels belonging to the $n=3$ and $n=4$ configurations, the relative difference between our two calculations for each level is shown in Table 1. The average absolute difference with standard deviation of the present MBPT and MCDF energy values are $296 \pm 646 \mathrm{~cm}^{-1}$, corresponding to the average relative difference with the standard deviation of $0.001 \% \pm 0.002 \%$, which are satisfactory.

\subsection{Wavelengths, transition rates, and lifetimes}

Feldman et al. [4] identified 13 lines of the $2 s^{2} 2 p^{4}$ - $2 s 2 p^{5}$ transitions in a laser-produced plasma, and these lines have been compiled in the NIST ASD. In Table 4, the experimental wavelengths for these 13 lines are compared with the present MBPT and MCDF results. Transition rates from our MBPT and MCDF calculations are also compared with each other in the table. As shown in Table 4, the difference between the NIST and MBPT(MCDF) wavelength for each transition is within $0.1 \%$, which is quite satisfactory. Our MBPT and MCDF transition rates show good agreement with each other, the differences being within $5 \%$.

In the spirit of the uncertainty estimation method suggested by Kramida [49, 50, 51], the difference $\delta S$ of line strengths $S$ from the MBPT and MCDF calculations for each E1 transition is defined as $\delta S$ $=\left|S_{\mathrm{MCDF}}-S_{\mathrm{MBPT}}\right| / \max \left(S_{\mathrm{MCDF}}, S_{\mathrm{MBPT}}\right)$. The averaged uncertainties $\delta S_{a v}$ for line strengths $S$ from the present MBPT and MCDF calculations for E1 transitions in various ranges of $S$ are assessed to $1.7 \%$ for $S \geq 10^{-1} ; 4.1 \%$ for $10^{-1}>S \geq 10^{-2} ; 6.1 \%$ for $10^{-2}>S \geq 10^{-3} ; 8.7 \%$ for $10^{-3}>S \geq 10^{-4} ; 18 \%$ for $10^{-4}>S \geq 10^{-5}$, and $35 \%$ for $10^{-5}>S \geq 10^{-6}$. Then, the larger of $\delta S_{i j}$ and $\delta S_{a v}$ is accepted as the uncertainty of each particular line strength. About $1.4 \%$ have uncertainties of $\leq 2 \%$ (the category $\mathrm{A}+$ ), $1.0 \%$ have uncertainties of $\leq 3 \%$ (the category $\mathrm{A}$ ), $20.0 \%$ have uncertainties of $\leq 7 \%$ (the category $\mathrm{B}+$ ), $23.3 \%$ have uncertainties of $\leq 10 \%$ (the category B), $8.9 \%$ have uncertainties of $\leq 18 \%$ (the 
category $\mathrm{C}+$ ), $19.6 \%$ have uncertainties of $\leq 25 \%$ (the category $\mathrm{C}$ ), and $13.5 \%$ have uncertainties of $\leq$ $40 \%$ (the category $\mathrm{D}+$ ), while $12.3 \%$ have uncertainties of $>40 \%$ (categories $\mathrm{D}$ and $\mathrm{E}$ ).

Again, using the method suggested in [50, 51], the uncertainties of the $S$ values for M1 and E2 transitions are estimated. The estimated uncertainties for all $\mathrm{E} 1, \mathrm{M} 1$, and $\mathrm{E} 2$ transitions with $\mathrm{BF} \geq 10^{-5}$ are listed in Table 2,

Our MBPT and MCDF lifetimes for the lowest 318 levels of the $n \leq 4$ configurations in O-like Mo XXXV, which are calculated by considering all possible E1, M1, and E2 transitions, are listed in Table 1. For the lowest 10 levels of the $n=2$ complex, the MCDF lifetimes agree very well with the MBPT results, and the differences are within $3 \%$. For the remaining 308 levels belonging to the $n=3$ and $n=4$ configurations, the average difference with the standard deviation is $0.5 \% \pm 4.2 \%$.

\section{Conclusions}

Employing two state-of-the-art methods (MBPT and MCDF), excitation energies and lifetimes of the lowest 318 levels for the $n \leq 4$ configurations have been calculated for O-like Mo XXXV. Wavelengths, $\mathrm{E} 1, \mathrm{M} 1$, and E2 transition rates, line strengths, and oscillator strengths for the transitions among these 318 levels are also reported.

The accuracy of energy levels and transition probabilities is estimated by comparing the MBPT and MCDF results with available experimental and theoretical data. The average difference with the standard deviation with the NIST values for the $n=2$ levels are $-249 \pm 748 \mathrm{~cm}^{-1}$ for MBPT and $-68 \pm 471$ $\mathrm{cm}^{-1}$ for MCDF, which indicates the high accuracy of the present two calculations. For the $n=3,4$ levels, the average absolute difference of the present MBPT and MCDF energy values is $296 \pm 646 \mathrm{~cm}^{-1}$, corresponding to the average relative difference of $0.001 \% \pm 0.002 \%$, where the standard deviations are indicated after the values. The uncertainty of the line strength is assessed for each transition, and is available in Table 2. The present calculations provide a consistent and accurate data set for line identification and modeling purposes, which can also be considered as a benchmark for other calculations.

\section{Acknowledges}

We acknowledge the support from the National Key Research and Development Program of China under Grant No. 2017YFA0403200, the Science Challenge Project of China Academy of Engineering Physics (CAEP) under Grant No. TZ2016005, the National Natural Science Foundation of China (Grant No. 11703004, No. 11674066, No. 11504421, and No. 11474034), the Natural Science Foundation of Hebei Province, China (A2019201300 and A2017201165), and the Natural Science Foundation of Educational Department of Hebei Province, China (BJ2018058). Kai Wang expresses his gratitude to the support from the visiting researcher program of Fudan University. 


\section{References}

[1] M. W. D. Mansfield, N. J. Peacock, C. C. Smith, M. G. Hobby, R. D. Cowan, Journal of Physics B Atomic Molecular Physics 11 (1978) 1521-1544.

[2] J. Reader, A. Tauheed, Journal of Physics B Atomic Molecular Physics 48 (2015) 144001.

[3] R. Lokasani, G. Arai, Y. Kondo, H. Hara, T.-H. Dinh, T. Ejima, T. Hatano, W. Jiang, T. Makimura, B. Li, P. Dunne, G. O’Sullivan, T. Higashiguchi, J. Limpouch, Applied Physics Letters 109 (2016) 194103.

[4] U. Feldman, J. O. Ekberg, J. F. Seely, C. M. Brown, D. R. Kania, B. J. MacGowan, C. J. Keane, W. E. Behring, Journal of the Optical Society of America B Optical Physics 8 (1991) 531-537.

[5] C. J. Fontes, H. L. Zhang, Atomic Data and Nuclear Data Tables 101 (2015) 143-157.

[6] F. Hu, J. Yang, C. Wang, G. Jiang, X. Zhao, H. Zang, Open Physics 9 (2011) 1228-1236.

[7] M. F. Gu, Atomic Data and Nuclear Data Tables 89 (2005) 267 - 293.

[8] H. L. Zhang, D. H. Sampson, Atomic Data and Nuclear Data Tables 82 (2002) 357-389.

[9] M. J. Vilkas, Y. Ishikawa, K. Koc, Physical Review A 60 (1999) 2808-2821.

[10] J. E. Rice, K. B. Fournier, J. A. Goetz, E. S. Marmar, J. L. Terry, J. Phys. B: At. Mol. Opt. Phys. 33 (2000) $5435-5462$.

[11] I. Kink, J. M. Laming, E. Takács, J. V. Porto, J. D. Gillaspy, E. Silver, H. Schnopper, S. R. Bandler, M. Barbera, N. Brickhouse, S. Murray, N. Madden, D. Landis, J. Beeman, E. E. Haller, Phys. Rev. E 63 (2001) 046409.

[12] K. Wang, P. Jönsson, J. Ekman, G. Gaigalas, M. R. Godefroid, R. Si, Z. B. Chen, S. Li, C. Y. Chen, J. Yan, The Astrophysical Journal Supplement Series 229 (2017) 37.

[13] K. Wang, P. Jönsson, J. Ekman, R. Si, Z. Chen, Y. Li, C. Chen, J. Yan, Journal of Quantitative Spectroscopy and Radiative Transfer 194 (2017) 108-112.

[14] I. Lindgren, J. Phys. B: At. Mol. Opt. Phys. 7 (1974) 2441.

[15] M. S. Safronova, W. R. Johnson, U. I. Safronova, Physical Review A 53 (1996) 4036-4053.

[16] M. F. Gu, The Astrophysical Journal Supplement Series 156 (2005) 105.

[17] M. F. Gu, The Astrophysical Journal Supplement Series 169 (2007) 154.

[18] I. P. Grant, Relativistic Quantum Theory of Atoms and Molecules, 2007. doi $10.1007 / 978-0-387-35069-1$.

[19] C. Froese Fischer, M. Godefroid, T. Brage, P. Jönsson, G. Gaigalas, J. Phys. B: At. Mol. Opt. Phys. 49 (2016) 182004.

[20] M. F. Gu, Canadian Journal of Physics 86 (2008) 675-689.

[21] P. Jönsson, X. He, C. Froese Fischer, I. Grant, Computer Physics Communications 177 (2007) 597 - 622.

[22] P. Jönsson, G. Gaigalas, J. Bieroń, C. Froese Fischer, I. P. Grant, Computer Physics Communications 184 (2013) $2197-2203$.

[23] K. Wang, D. F. Li, H. T. Liu, X. Y. Han, B. Duan, C. Y. Li, J. G. Li, X. L. Guo, C. Y. Chen, J. Yan, The Astrophysical Journal Supplement Series 215 (2014) 26.

[24] K. Wang, X. L. Guo, H. T. Liu, D. F. Li, F. Y. Long, X. Y. Han, B. Duan, J. G. Li, M. Huang, Y. S. Wang, R. Hutton, Y. M. Zou, J. L. Zeng, C. Y. Chen, J. Yan, The Astrophysical Journal Supplement Series 218 (2015) 16.

[25] K. Wang, R. Si, W. Dang, P. Jönsson, X. L. Guo, S. Li, Z. B. Chen, H. Zhang, F. Y. Long, H. T. Liu, D. F. Li, R. Hutton, C. Y. Chen, J. Yan, The Astrophysical Journal Supplement Series 223 (2016) 3.

[26] K. Wang, Z. B. Chen, R. Si, P. Jönsson, J. Ekman, X. L. Guo, S. Li, F. Y. Long, W. Dang, X. H. Zhao, R. Hutton, C. Y. Chen, J. Yan, X. Yang, The Astrophysical Journal Supplement Series 226 (2016) 14.

[27] K. Wang, P. Jönsson, J. Ekman, T. Brage, C. Y. Chen, C. Froese Fischer, G. Gaigalas, M. Godefroid, Physical Review Letters 119 (2017) 189301.

[28] K. Wang, S. Li, P. Jönsson, N. Fu, W. Dang, X. Guo, C. Chen, J. Yan, Z. Chen, R. Si, Journal of Quantitative Spectroscopy and Radiative Transfer 187 (2017) 375 - 402.

[29] K. Wang, P. Jönsson, G. Gaigalas, L. Radžiūtė, P. Rynkun, G. Del Zanna, C. Y. Chen, The Astrophysical Journal Supplement Series 235 (2018) 27.

[30] K. Wang, C. X. Song, P. Jönsson, G. Del Zanna, S. Schiffmann, M. Godefroid, G. Gaigalas, X. H. Zhao, R. Si, C. Y. Chen, J. Yan, The Astrophysical Journal Supplement Series 239 (2018) 30.

[31] K. Wang, Z. B. Chen, C. Y. Zhang, R. Si, P. Jönsson, H. Hartman, M. F. Gu, C. Y. Chen, J. Yan, The Astrophysical Journal Supplement Series 234 (2018) 40.

[32] K. Wang, C. Zhang, P. Jönsson, R. Si, X. Zhao, Z. Chen, X. Guo, C. Chen, J. Yan, Journal of Quantitative Spectroscopy and Radiative Transfer 208 (2018) 134-151.

[33] K. Wang, C. X. Song, P. Jnsson, J. Ekman, M. Godefroid, C. Y. Zhang, R. Si, X. H. Zhao, C. Y. Chen, J. Yan, The Astrophysical Journal 864 (2018) 127.

[34] K. Wang, Y. Wang, C. Y. Zhang, X. H. Zhao, Z. B. Chen, R. Si, C. Y. Chen, J. Yan, Journal of Quantitative Spectroscopy and Radiative Transfer 220 (2018) 5-27.

[35] Z. B. Chen, K. Ma, H. J. Wang, K. Wang, X. B. Liu, J. L. Zeng, Atomic Data and Nuclear Data Tables 113 (2017) $258-292$.

[36] Z. B. Chen, X. L. Guo, K. Wang, Journal of Quantitative Spectroscopy and Radiative Transfer 206 (2018) $213-232$.

[37] Z. B. Chen, K. Wang, Journal of Quantitative Spectroscopy and Radiative Transfer 234 (2019) 90-97. 
[38] Z. Chen, C. Sang, K. Wang, Journal of Quantitative Spectroscopy and Radiative Transfer 225 (2019) 76-83.

[39] X. L. Guo, M. Huang, J. Yan, S. Li, R. Si, C. Y. Li, C. Y. Chen, Y. S. Wang, Y. M. Zou, Journal of Physics B Atomic Molecular Physics 48 (2015) 144020.

[40] X. L. Guo, R. Si, S. Li, M. Huang, R. Hutton, Y. S. Wang, C. Y. Chen, Y. M. Zou, K. Wang, J. Yan, C. Y. Li, T. Brage, Physical Review A 93 (2016) 012513.

[41] R. Si, S. Li, X. L. Guo, Z. B. Chen, T. Brage, P. Jönsson, K. Wang, J. Yan, C. Y. Chen, Y. M. Zou, The Astrophysical Journal Supplement Series 227 (2016) 16.

[42] R. Si, C. Zhang, Y. Liu, Z. Chen, X. Guo, S. Li, J. Yan, C. Chen, K. Wang, Journal of Quantitative Spectroscopy and Radiative Transfer 189 (2017) 249-257.

[43] R. Si, C. Y. Zhang, Z. Y. Cheng, K. Wang, P. Jönsson, K. Yao, M. F. Gu, C. Y. Chen, The Astrophysical Journal Supplement Series 239 (2018) 3.

[44] Z. Zhao, K. Wang, S. Li, R. Si, C. Chen, Z. Chen, J. Yan, Yu. Ralchenko, Atomic Data and Nuclear Data Tables 119 (2018).

[45] L. Sturesson, P. Jönsson, C. Froese Fischer, Computer Physics Communications 177 (2007) 539-550.

[46] A. Kramida, Yu. Ralchenko, J. Reader, and NIST ASD Team, NIST Atomic Spectra Database (ver. 5.6.1), [Online]. Available: https://physics.nist.gov/asd [2019, May 27]. National Institute of Standards and Technology, Gaithersburg, MD., 2018.

[47] G. Gaigalas, T. Zalandauskas, S. Fritzsche, Computer Physics Communications 157 (2004) 239-253.

[48] G. Gaigalas, C. Froese Fischer, P. Rynkun, P. Jönsson, Atoms 5 (2017) 6.

[49] A. Kramida, Fusion Science and Technology 63 (2013) 313-323.

[50] A. Kramida, Atoms 2 (2014) 86-122.

[51] A. Kramida, The Astrophysical Journal Supplement Series 212 (2014) 11. 
Table 1. Excitation energies $\left(E\right.$ in $\left.\mathrm{cm}^{-1}\right)$ and lifetimes $\left(\tau\right.$ in $\left.\mathrm{s}^{-1}\right)$ for the lowest 318 levels arising from the $2 s^{2} 2 p^{4}, 2 s 2 p^{5}, 2 p^{6}$, $2 s^{2} 2 p^{3} 3 l, 2 s 2 p^{4} 3 l, 2 p^{5} 3 l$, and $2 s^{2} 2 p^{3} 4 l$ configurations of O-like Mo XXXV from the present MBPT and MCDF calculations. $J \pi$ : the total quantum number $J$ and parity $\pi ; \Delta E$ - the differences (in $\mathrm{cm}^{-1}$ ) between the MBPT and MCDF calculated excitation energies.

\begin{tabular}{|c|c|c|c|c|c|c|c|c|}
\hline Key & $L S J$-coupled CSF & $J \pi$ & $E_{\mathrm{MCDF}}$ & $E_{\mathrm{MBPT}}$ & $\Delta E$ & $\tau_{\mathrm{MCDF}}$ & $\tau_{\mathrm{MBPT}}$ & $j j$-coupled $\mathrm{CSF}^{a, b, c}$ \\
\hline 1 & $2 s^{2} 2 p^{4}\left({ }^{3} P\right){ }^{3} P_{2}$ & $2+$ & 0 & 0 & 0 & & & $2 p+2(4) 4$ \\
\hline 2 & $2 s^{2} 2 p^{4}\left({ }^{1} S\right){ }^{1} S_{0}$ & $0+$ & 207772 & 207830 & 57 & 7.987E-02 & 7.987E-02 & $2 p+2(0) 0$ \\
\hline 3 & $2 s^{2} 2 p^{4}\left({ }^{3} P\right){ }^{3} P_{1}$ & $1+$ & 863686 & 863215 & -471 & $7.767 \mathrm{E}-08$ & $7.767 \mathrm{E}-08$ & $2 \mathrm{p}-1(1) 1.2 \mathrm{p}+3(3) 2$ \\
\hline 4 & $2 s^{2} 2 p^{4}\left({ }^{1} D\right){ }^{1} D_{2}$ & $2+$ & 976768 & 976432 & -337 & $1.414 \mathrm{E}-07$ & $1.414 \mathrm{E}-07$ & $2 \mathrm{p}-1(1) 1.2 \mathrm{p}+3(3) 4$ \\
\hline 5 & $2 s^{2} 2 p^{4}\left({ }^{3} P\right){ }^{3} P_{0}$ & $0+$ & 1921492 & 1920667 & -825 & $2.842 \mathrm{E}-08$ & $2.842 \mathrm{E}-08$ & $2 p+4(0) 0$ \\
\hline 6 & $2 s^{2} S 2 p^{53} P_{2}^{\circ}$ & $2-$ & 2379704 & 2380355 & 651 & $4.938 \mathrm{E}-12$ & $4.938 \mathrm{E}-12$ & $2 \mathrm{~s}+1(1) 1.2 \mathrm{p}+3(3) 4$ \\
\hline 7 & $2 s^{2} S 2 p^{5}{ }^{3} P_{1}^{\circ}$ & $1-$ & 2654158 & 2655091 & 933 & $2.294 \mathrm{E}-12$ & $2.294 \mathrm{E}-12$ & $2 s+1(1) 1.2 p+3(3) 2$ \\
\hline 8 & $2 s^{2} S 2 p^{5}{ }^{3} P_{0}^{\circ}$ & $0-$ & 3289601 & 3289830 & 228 & $3.061 \mathrm{E}-12$ & $3.061 \mathrm{E}-12$ & $2 \mathrm{~s}+1(1) 1.2 \mathrm{p}-1(1) 0$ \\
\hline 9 & $2 s^{2} S 2 p^{51} P_{1}^{\circ}$ & $1-$ & 3529720 & 3530442 & 722 & $1.845 \mathrm{E}-12$ & $1.845 \mathrm{E}-12$ & $2 \mathrm{~s}+1(1) 1.2 \mathrm{p}-1(1) 2$ \\
\hline 10 & $2 p^{6}{ }^{1} S_{0}$ & $0+$ & 5271717 & 5272387 & 670 & $1.321 \mathrm{E}-12$ & $1.321 \mathrm{E}-12$ & $2 p+4(0) 0$ \\
\hline 11 & $2 s^{2} 2 p^{3}\left({ }^{2} D\right){ }^{2} D 3 s^{3} D_{2}^{\circ}$ & $2-$ & 20650637 & 20651158 & 521 & $1.486 \mathrm{E}-13$ & $1.486 \mathrm{E}-13$ & $2 p+1(3) 3.3 s+1(1) 4$ \\
\hline 12 & $2 s^{2} 2 p^{3}\left({ }^{2} D\right){ }^{2} D 3 s^{3} D_{1}^{\circ}$ & $1-$ & 20696082 & 20696833 & 752 & $4.980 \mathrm{E}-14$ & $4.980 \mathrm{E}-14$ & $2 p+1(3) 3.3 s+1(1) 2$ \\
\hline 13 & $2 s^{2} 2 p^{3}\left({ }^{2} D\right)^{2} D 3 p^{3} D_{1}$ & $1+$ & 21088106 & 21087880 & -226 & $1.209 \mathrm{E}-11$ & $1.209 \mathrm{E}-11$ & $2 \mathrm{p}+1(3) 3.3 \mathrm{p}-1(1) 2$ \\
\hline 14 & $2 s^{2} 2 p^{3}\left({ }^{4} S\right){ }^{4} S 3 p^{3} P_{2}$ & $2+$ & 21100632 & 21100445 & -188 & $1.121 \mathrm{E}-11$ & $1.121 \mathrm{E}-11$ & $2 p+1(3) 3.3 p-1(1) 4$ \\
\hline 15 & $2 s^{2} 2 p^{3}\left(^{2} D\right)^{2} D 3 p^{1} F_{3}$ & $3+$ & 21339859 & 21339494 & -365 & $1.264 \mathrm{E}-11$ & $1.264 \mathrm{E}-11$ & $2 p+1(3) 3.3 p+1(3) 6$ \\
\hline 16 & $2 s^{2} 2 p^{3}\left({ }^{4} S\right){ }^{4} S 3 p^{3} P_{1}$ & $1+$ & 21342916 & 21342630 & -286 & $7.800 \mathrm{E}-12$ & $7.800 \mathrm{E}-12$ & $2 p+1(3) 3.3 p+1(3) 2$ \\
\hline 17 & $2 s^{2} 2 p^{3}\left({ }^{4} S\right){ }^{4} S 3 s^{5} S_{2}^{\circ}$ & $2-$ & 21442137 & 21442500 & 363 & $1.304 \mathrm{E}-13$ & $1.304 \mathrm{E}-13$ & $2 p-1(1) 1.2 p+2(4) 3.3 s+1(1) 4$ \\
\hline 18 & $2 s^{2} 2 p^{3}\left({ }^{2} D\right){ }^{2} D 3 p^{3} D_{2}$ & $2+$ & 21443360 & 21443353 & -7 & $1.076 \mathrm{E}-11$ & $1.076 \mathrm{E}-11$ & $2 p+1(3) 3.3 p+1(3) 4$ \\
\hline 19 & $2 s^{2} 2 p^{3}\left({ }^{4} S\right){ }^{4} S 3 s^{3} S_{1}^{\circ}$ & $1-$ & 21486265 & 21486867 & 603 & $4.736 \mathrm{E}-14$ & $4.736 \mathrm{E}-14$ & $2 \mathrm{p}-1(1) 1.2 \mathrm{p}+2(4) 3.3 \mathrm{~s}+1(1) 2$ \\
\hline 20 & $2 s^{2} 2 p^{3}\left({ }^{4} S\right){ }^{4} S 3 p^{3} P_{0}$ & $0+$ & 21539884 & 21540069 & 186 & $2.594 \mathrm{E}-12$ & $2.594 \mathrm{E}-12$ & $2 p+1(3) 3.3 p+1(3) 0$ \\
\hline 21 & $2 s^{2} 2 p^{3}\left({ }^{2} D\right){ }^{2} D 3 s^{3} D_{3}^{\circ}$ & $3-$ & 21572314 & 21572756 & 443 & 7.952E-14 & 7.952E-14 & $2 \mathrm{p}-1(1) 1.2 \mathrm{p}+2(4) 5.3 \mathrm{~s}+1(1) 6$ \\
\hline 22 & $2 s^{2} 2 p^{3}\left(^{2} D\right)^{2} D 3 s^{1} D_{0}^{\circ}$ & $2-$ & 21606547 & 21607149 & 602 & $5.000 \mathrm{E}-14$ & $5.000 \mathrm{E}-14$ & $2 \mathrm{p}-1(1) 1.2 \mathrm{p}+2(4) 5.3 \mathrm{~s}+1(1) 4$ \\
\hline 23 & $2 s^{2} 2 p^{3}\left({ }^{2} P\right)^{2} P 3 s^{3} P_{0}^{\circ}$ & $0-$ & 21744917 & 21745043 & 126 & $1.148 \mathrm{E}-13$ & $1.148 \mathrm{E}-13$ & $2 \mathrm{p}-1(1) 1.2 \mathrm{p}+2(0) 1.3 \mathrm{~s}+1(1) 0$ \\
\hline 24 & $2 s^{2} 2 p^{3}\left({ }^{2} P\right)^{2} P 3 s^{3} P_{1}^{\circ}$ & $1-$ & 21759710 & 21759924 & 214 & $5.902 \mathrm{E}-14$ & $5.902 \mathrm{E}-14$ & $2 \mathrm{p}-1(1) 1.2 \mathrm{p}+2(0) 1.3 \mathrm{~s}+1(1) 2$ \\
\hline 25 & $2 s^{2} 2 p^{3}\left({ }^{2} P\right)^{2} P 3 d^{3} D_{2}^{\circ}$ & $2-$ & 21839491 & 21840372 & 881 & $2.556 \mathrm{E}-13$ & $2.556 \mathrm{E}-13$ & $2 p+1(3) 3.3 d-1(3) 4$ \\
\hline 26 & $2 s^{2} 2 p^{3}\left({ }^{2} D\right){ }^{2} D 3 d^{3} G_{3}^{\circ}$ & $3-$ & 21862744 & 21863661 & 917 & $9.965 \mathrm{E}-13$ & $9.965 \mathrm{E}-13$ & $2 p+1(3) 3.3 d-1(3) 6$ \\
\hline 27 & $2 s^{2} 2 p^{3}\left({ }^{4} S\right){ }^{4} S 3 p^{5} P_{1}$ & $1+$ & 21870635 & 21870247 & -388 & $1.675 \mathrm{E}-11$ & $1.675 \mathrm{E}-11$ & $2 p-1(1) 1.2 p+2(4) 3.3 p-1(1) 2$ \\
\hline 28 & $2 s^{2} 2 p^{3}\left({ }^{4} S\right){ }^{4} S 3 d^{5} D_{1}^{\circ}$ & $1-$ & 21879333 & 21880156 & 823 & $3.845 \mathrm{E}-14$ & $3.845 \mathrm{E}-14$ & $2 \mathrm{p}+1(3) 3.3 \mathrm{~d}-1(3) 2$ \\
\hline 29 & $2 s^{2} 2 p^{3}\left({ }^{2} D\right){ }^{2} D 3 d^{1} S_{0}^{\circ}$ & $0-$ & 21881341 & 21882166 & 825 & $1.763 \mathrm{E}-12$ & $1.763 \mathrm{E}-12$ & $2 p+1(3) 3.3 d-1(3) 0$ \\
\hline 30 & $2 s^{2} 2 p^{3}\left({ }^{2} D\right){ }^{2} D 3 d^{1} G_{4}^{\circ}$ & $4-$ & 21916177 & 21917059 & 882 & $7.989 \mathrm{E}-11$ & $7.989 \mathrm{E}-11$ & $2 \mathrm{p}+1(3) 3.3 \mathrm{~d}+1(5) 8$ \\
\hline 31 & $2 s^{2} 2 p^{3}\left({ }^{2} D\right)^{2} D 3 p^{3} F_{2}$ & $2+$ & 21918698 & 21918410 & -288 & $2.298 \mathrm{E}-11$ & $2.298 \mathrm{E}-11$ & $2 \mathrm{p}-1(1) 1.2 \mathrm{p}+2(4) 3.3 \mathrm{p}-1(1) 4$ \\
\hline 32 & $2 s^{2} 2 p^{3}\left({ }^{4} S\right){ }^{4} S 3 d^{5} D_{2}^{\circ}$ & $2-$ & 21962255 & 21963172 & 916 & $1.412 \mathrm{E}-14$ & $1.412 \mathrm{E}-14$ & $2 \mathrm{p}+1(3) 3.3 \mathrm{~d}+1(5) 4$ \\
\hline 33 & $2 s^{2} 2 p^{3}\left({ }^{2} D\right){ }^{2} D 3 p^{3} F_{3}$ & $3+$ & 22015588 & 22015303 & -285 & $1.645 \mathrm{E}-11$ & $1.645 \mathrm{E}-11$ & $2 \mathrm{p}-1(1) 1.2 \mathrm{p}+2(4) 5.3 \mathrm{p}-1(1) 6$ \\
\hline 34 & $2 s^{2} 2 p^{3}\left({ }^{2} D\right)^{2} D 3 d^{3} F_{3}^{\circ}$ & $3-$ & 22044284 & 22045384 & 1101 & $6.373 \mathrm{E}-15$ & $6.373 \mathrm{E}-15$ & $2 p+1(3) 3.3 d+1(5) 6$ \\
\hline 35 & $2 s^{2} 2 p^{3}\left({ }^{4} S\right){ }^{4} S 3 d^{3} D_{1}^{\circ}$ & $1-$ & 22071986 & 22073049 & 1063 & $9.081 \mathrm{E}-15$ & $9.081 \mathrm{E}-15$ & $2 \mathrm{p}+1(3) 3.3 \mathrm{~d}+1(5) 2$ \\
\hline 36 & $2 s^{2} 2 p^{3}\left({ }^{4} S\right){ }^{4} S 3 p^{5} P_{3}$ & $3+$ & 22126012 & 22125475 & -537 & $1.060 \mathrm{E}-11$ & $1.060 \mathrm{E}-11$ & $2 p-1(1) 1.2 p+2(4) 3.3 p+1(3) 6$ \\
\hline 37 & $2 s^{2} 2 p^{3}\left({ }^{4} S\right){ }^{4} S 3 p^{5} P_{2}$ & $2+$ & 22127625 & 22127143 & -482 & $5.112 \mathrm{E}-12$ & $5.112 \mathrm{E}-12$ & $2 \mathrm{p}-1(1) 1.2 \mathrm{p}+2(4) 3.3 \mathrm{p}+1(3) 4$ \\
\hline 38 & $2 s^{2} 2 p^{3}\left({ }^{2} D\right)^{2} D 3 p^{3} P_{0}$ & $0+$ & 22138613 & 22138217 & -395 & $6.649 \mathrm{E}-12$ & $6.649 \mathrm{E}-12$ & $2 \mathrm{p}-1(1) 1.2 \mathrm{p}+2(4) 3.3 \mathrm{p}+1(3) 0$ \\
\hline 39 & $2 s^{2} 2 p^{3}\left({ }^{2} D\right)^{2} D 3 p^{3} P_{2}$ & $2+$ & 22148113 & 22148354 & 241 & $2.477 \mathrm{E}-12$ & $2.477 \mathrm{E}-12$ & $2 \mathrm{p}-1(1) 1.2 \mathrm{p}+2(4) 5.3 \mathrm{p}-1(1) 4$ \\
\hline 40 & $2 s^{2} 2 p^{3}\left({ }^{2} P\right)^{2} P 3 p^{3} D_{1}$ & $1+$ & 22149046 & 22148482 & -565 & $9.339 \mathrm{E}-12$ & $9.339 \mathrm{E}-12$ & $2 \mathrm{p}-1(1) 1.2 \mathrm{p}+2(0) 1.3 \mathrm{p}-1(1) 2$ \\
\hline 41 & $2 s^{2} 2 p^{3}\left({ }^{2} D\right){ }^{2} D 3 p p^{1} P_{1}$ & $1+$ & 22244154 & 22243774 & -380 & $6.644 \mathrm{E}-12$ & $6.644 \mathrm{E}-12$ & $2 p-1(1) 1.2 p+2(4) 3.3 p+1(3) 2$ \\
\hline 42 & $2 s^{2} 2 p^{3}\left({ }^{2} D\right){ }^{2} D 3 p^{3} D_{3}$ & $3+$ & 22252714 & 22252265 & -450 & $1.188 \mathrm{E}-11$ & $1.188 \mathrm{E}-11$ & $2 \mathrm{p}-1(1) 1.2 \mathrm{p}+2(4) 5.3 \mathrm{p}+1(3) 6$ \\
\hline 43 & $2 s^{2} 2 p^{3}\left({ }^{2} D\right){ }^{2} D 3 p^{3} F_{4}$ & $4+$ & 22263500 & 22263072 & -427 & $1.520 \mathrm{E}-11$ & $1.520 \mathrm{E}-11$ & $2 \mathrm{p}-1(1) 1.2 \mathrm{p}+2(4) 5.3 \mathrm{p}+1(3) 8$ \\
\hline 44 & $2 s^{2} 2 p^{3}\left({ }^{2} D\right)^{2} D 3 p^{3} P_{1}$ & $1+$ & 22350764 & 22350673 & -91 & $1.665 \mathrm{E}-12$ & $1.665 \mathrm{E}-12$ & $2 \mathrm{p}-1(1) 1.2 \mathrm{p}+2(4) 5.3 \mathrm{p}+1(3) 2$ \\
\hline 45 & $2 s^{2} 2 p^{3}\left({ }^{2} P\right)^{2} P 3 p^{3} P_{0}$ & $0+$ & 22358146 & 22358194 & 47 & $2.632 \mathrm{E}-12$ & $2.632 \mathrm{E}-12$ & $2 \mathrm{p}-1(1) 1.2 \mathrm{p}+2(0) 1.3 \mathrm{p}-1(1) 0$ \\
\hline 46 & $2 s^{2} 2 p^{3}\left({ }^{2} D\right){ }^{2} D 3 p^{1} D_{2}$ & $2+$ & 22421438 & 22421218 & -220 & $1.940 \mathrm{E}-12$ & $1.940 \mathrm{E}-12$ & $2 \mathrm{p}-1(1) 1.2 \mathrm{p}+2(4) 5.3 \mathrm{p}+1(3) 4$ \\
\hline 47 & $2 s^{2} 2 p^{3}\left({ }^{2} P\right)^{2} P 3 p^{3} P_{1}$ & $1+$ & 22462553 & 22462031 & -522 & $2.220 \mathrm{E}-12$ & $2.220 \mathrm{E}-12$ & $2 \mathrm{p}-1(1) 1.2 \mathrm{p}+2(0) 1.3 \mathrm{p}+1(3) 2$ \\
\hline 48 & $2 s^{2} 2 p^{3}\left({ }^{2} P\right)^{2} P 3 p^{3} D_{2}$ & $2+$ & 22486651 & 22486365 & -286 & $2.754 \mathrm{E}-12$ & $2.754 \mathrm{E}-12$ & $2 \mathrm{p}-1(1) 1.2 \mathrm{p}+2(0) 1.3 \mathrm{p}+1(3) 4$ \\
\hline 49 & $2 s^{2} 2 p^{3}\left({ }^{2} P\right)^{2} P 3 s^{3} P_{2}^{\circ}$ & $2-$ & 22538636 & 22538645 & 9 & $5.859 \mathrm{E}-14$ & $5.859 \mathrm{E}-14$ & $2 p+3(3) 3.3 s+1(1) 4$ \\
\hline 50 & $2 s^{2} 2 p^{3}\left({ }^{2} P\right)^{2} P 3 s^{1} P_{1}^{\circ}$ & $1-$ & 22568066 & 22568222 & 156 & $4.521 \mathrm{E}-14$ & $4.521 \mathrm{E}-14$ & $2 p+3(3) 3.3 s+1(1) 2$ \\
\hline 51 & $2 s^{2} 2 p^{3}\left({ }^{2} D\right)^{2} D 3 d^{3} D_{1}^{\circ}$ & $1-$ & 22648460 & 22649188 & 727 & $6.105 \mathrm{E}-14$ & $6.105 \mathrm{E}-14$ & $2 \mathrm{p}-1(1) 1.2 \mathrm{p}+2(4) 3.3 \mathrm{~d}-1(3) 2$ \\
\hline 52 & $2 s^{2} 2 p^{3}\left({ }^{2} D\right){ }^{2} D 3 d^{3} F_{2}^{\circ}$ & $2-$ & 22655526 & 22656275 & 749 & $5.151 \mathrm{E}-14$ & $5.151 \mathrm{E}-14$ & $2 p-1(1) 1.2 p+2(4) 3.3 d-1(3) 4$ \\
\hline 53 & $2 s^{2} 2 p^{3}\left({ }^{4} S\right){ }^{4} S 3 d^{5} D_{0}^{\circ}$ & $0-$ & 22659553 & 22660287 & 734 & $7.713 \mathrm{E}-14$ & $7.713 \mathrm{E}-14$ & $2 \mathrm{p}-1(1) 1.2 \mathrm{p}+2(4) 3.3 \mathrm{~d}-1(3) 0$ \\
\hline 54 & $2 s^{2} 2 p^{3}\left({ }^{4} S\right){ }^{4} S 3 d^{5} D_{3}^{\circ}$ & $3-$ & 22685184 & 22685956 & 772 & $3.907 \mathrm{E}-14$ & $3.907 \mathrm{E}-14$ & $2 \mathrm{p}-1(1) 1.2 \mathrm{p}+2(4) 3.3 \mathrm{~d}-1(3) 6$ \\
\hline 55 & $2 s^{2} 2 p^{3}\left({ }^{4} S\right){ }^{4} S 3 d^{5} D_{4}^{\circ}$ & $4-$ & 22704297 & 22705079 & 782 & $8.306 \mathrm{E}-11$ & $8.306 \mathrm{E}-11$ & $2 \mathrm{p}-1(1) 1.2 \mathrm{p}+2(4) 3.3 \mathrm{~d}+1(5) 8$ \\
\hline 56 & $2 s^{2} 2 p^{3}\left({ }^{4} S\right){ }^{4} S 3 d^{3} D^{\circ}$ & $3-$ & 22712027 & 22712898 & 871 & $9.071 \mathrm{E}-14$ & $9.071 \mathrm{E}-14$ & $2 \mathrm{p}-1(1) 1.2 \mathrm{p}+2(4) 3.3 \mathrm{~d}+1(5) 6$ \\
\hline 57 & $2 s^{2} S 2 p^{4}\left({ }^{3} P\right){ }^{4} P 3 s^{5} P_{3}$ & $3+$ & 22715626 & 22714630 & -996 & $5.899 \mathrm{E}-13$ & $5.899 \mathrm{E}-13$ & $2 s+1(1) 1.2 p+2(4) 5.3 s+1(1) 6$ \\
\hline 58 & $2 s^{2} 2 p^{3}\left({ }^{2} D\right){ }^{2} D 3 d^{3} G_{4}^{\circ}$ & $4-$ & 22783461 & 22784420 & 959 & $4.242 \mathrm{E}-11$ & $4.242 \mathrm{E}-11$ & $2 \mathrm{p}-1(1) 1.2 \mathrm{p}+2(4) 5.3 \mathrm{~d}-1(3) 8$ \\
\hline 59 & $2 s^{2} 2 p^{3}\left({ }^{2} D\right){ }^{2} D 3 d^{1} P^{\circ}$ & $1-$ & 22790735 & 22791579 & 844 & $1.430 \mathrm{E}-14$ & $1.430 \mathrm{E}-14$ & $2 \mathrm{p}-1(1) 1.2 \mathrm{p}+2(4) 3.3 \mathrm{~d}+1(5) 2$ \\
\hline 60 & $2 s^{2} 2 p^{3}\left({ }^{2} D\right)^{2} D 3 d^{3} P^{1}$ & $2-$ & 22812793 & 22813601 & 807 & $8.916 \mathrm{E}-15$ & $8.916 \mathrm{E}-15$ & $2 \mathrm{p}-1(1) 1.2 \mathrm{p}+2(4) 3.3 \mathrm{~d}+1(5) 4$ \\
\hline 61 & $2 s^{2} S 2 p^{4}\left({ }^{3} P\right){ }^{4} P 3 s^{3} P_{2}^{2}$ & $2+$ & 22829810 & 22829389 & -421 & $6.458 \mathrm{E}-14$ & $6.458 \mathrm{E}-14$ & $2 s+1(1) 1.2 p+2(4) 5.3 s+1(1) 4$ \\
\hline
\end{tabular}


Table 1. (continued)

\begin{tabular}{|c|c|c|c|c|c|c|c|c|}
\hline Key & $L S J$-coupled CSF & $J \pi$ & $E_{\mathrm{MCDF}}$ & $E_{\mathrm{MBPT}}$ & $\Delta E$ & $\tau_{\mathrm{MCDF}}$ & $\tau_{\mathrm{MBPT}}$ & $j j$-coupled $\mathrm{CSF}^{a, b, c}$ \\
\hline 62 & $2 p^{3}\left({ }^{2} D\right)^{2} D 3 d^{3} F_{4}^{\circ}$ & $4-$ & 830158 & 2831043 & 885 & 7.617E-11 & 7.617E-11 & $2 \mathrm{p}-1(1) 1.2 \mathrm{p}+2(4) 5.3 \mathrm{~d}+1(5) 8$ \\
\hline 63 & $2 s^{2} 2 p^{3}\left({ }^{2} D\right)^{2} D 3 d^{3} D_{3}^{\circ}$ & $3-$ & 22837163 & 22838068 & 905 & $6.548 \mathrm{E}-15$ & $6.548 \mathrm{E}-15$ & $2 \mathrm{p}-1(1) 1.2 \mathrm{p}+2(4) 5.3 \mathrm{~d}-1(3) 6$ \\
\hline 64 & $2 s^{2} 2 p^{3}\left({ }^{2} D\right){ }^{2} D 3 d^{3} G_{5}^{\circ}$ & $5-$ & 22840848 & 22841781 & 933 & $8.437 \mathrm{E}-11$ & $8.437 \mathrm{E}-11$ & $2 \mathrm{p}-1(1) 1.2 \mathrm{p}+2(4) 5.3 \mathrm{~d}+1(5) 10$ \\
\hline 65 & $2 s^{2} 2 p^{3}\left({ }^{2} D\right){ }^{2} D 3 d^{3} P_{0}^{\circ}$ & $0-$ & 22878419 & 22879263 & 844 & $1.369 \mathrm{E}-14$ & $1.369 \mathrm{E}-14$ & $2 \mathrm{p}-1(1) 1.2 \mathrm{p}+2(4) 5.3 \mathrm{~d}+1(5) 0$ \\
\hline 66 & $2 s^{2} 2 p^{3}\left({ }^{2} D\right)^{2} D 3 d^{3} P_{1}^{\circ}$ & $1-$ & 22881477 & 22882356 & 879 & $6.387 \mathrm{E}-15$ & $6.387 \mathrm{E}-15$ & $2 \mathrm{p}-1(1) 1.2 \mathrm{p}+2(4) 5.3 \mathrm{~d}-1(3) 2$ \\
\hline 67 & $2 s^{2} 2 p^{3}\left({ }^{2} D\right){ }^{2} D 3 d^{3} D_{2}^{\circ}$ & $2-$ & 22885462 & 22886390 & 928 & $6.273 \mathrm{E}-15$ & $6.273 \mathrm{E}-15$ & $2 \mathrm{p}-1(1) 1.2 \mathrm{p}+2(4) 5.3 \mathrm{~d}-1(3) 4$ \\
\hline 68 & $2 s^{2} 2 p^{3}\left({ }^{2} P\right){ }^{2} P 3 d{ }^{1} D_{2}^{\circ}$ & $2-$ & 22930758 & 22931410 & 652 & $2.118 \mathrm{E}-14$ & $2.118 \mathrm{E}-14$ & $2 \mathrm{p}-1(1) 1.2 \mathrm{p}+2(0) 1.3 \mathrm{~d}-1(3) 4$ \\
\hline 69 & $2 s^{2} 2 p^{3}\left({ }^{2} D\right)^{2} D 3 d^{3} S^{\circ}$ & $1-$ & 22940955 & 22941641 & 686 & $6.400 \mathrm{E}-15$ & $6.400 \mathrm{E}-15$ & $2 \mathrm{p}-1(1) 1.2 \mathrm{p}+2(4) 5.3 \mathrm{~d}+1(5) 2$ \\
\hline 70 & $2 s^{2} 2 p^{3}\left({ }^{2} D\right){ }^{2} D 3 d^{1} F_{3}^{\circ}$ & $3-$ & 22977582 & 22978659 & 1077 & $9.410 \mathrm{E}-15$ & $9.410 \mathrm{E}-15$ & $2 \mathrm{p}-1(1) 1.2 \mathrm{p}+2(4) 5.3 \mathrm{~d}+1(5) 6$ \\
\hline 71 & $2 s^{2} 2 p^{3}\left({ }^{2} P\right)^{2} P 3 d^{3} F_{2}^{0}$ & $2-$ & 23012320 & 23013008 & 688 & $1.064 \mathrm{E}-14$ & $1.064 \mathrm{E}-14$ & $2 \mathrm{p}-1(1) 1.2 \mathrm{p}+2(4) 5.3 \mathrm{~d}+1(5) 4$ \\
\hline 72 & $2 s^{2} 2 p^{3}\left({ }^{2} P\right)^{2} P 3 p^{3} S_{1}^{2}$ & $1+$ & 23036093 & 23035602 & -491 & $1.174 \mathrm{E}-12$ & $1.174 \mathrm{E}-12$ & $2 \mathrm{p}+3(3) 3.3 \mathrm{p}-1(1) 2$ \\
\hline 73 & $2 s^{2} 2 p^{3}\left({ }^{2} P\right)^{2} P 3 d^{3} P_{2}^{\circ}$ & $2-$ & 23063727 & 23064263 & 536 & $6.713 \mathrm{E}-15$ & $6.713 \mathrm{E}-15$ & $2 \mathrm{p}-1(1) 1.2 \mathrm{p}+2(0) 1.3 \mathrm{~d}+1(5) 4$ \\
\hline 74 & $2 s^{2} 2 p^{3}\left({ }^{2} P\right)^{2} P 3 p^{1} D_{2}$ & $2+$ & 23069439 & 23069193 & -246 & $8.834 \mathrm{E}-12$ & $8.834 \mathrm{E}-12$ & $2 p+3(3) 3.3 p-1(1) 4$ \\
\hline 75 & $2 s^{2} 2 p^{3}\left({ }^{2} P\right)^{2} P 3 d^{3} F_{3}^{\circ}$ & $3-$ & 23069895 & 23070507 & 613 & $9.205 \mathrm{E}-15$ & $9.205 \mathrm{E}-15$ & $2 \mathrm{p}-1(1) 1.2 \mathrm{p}+2(0) 1.3 \mathrm{~d}+1(5) 6$ \\
\hline 76 & $2 s^{2} 2 p^{3}\left({ }^{2} P\right)^{2} P 3 d^{3} D_{1}^{\circ}$ & $1-$ & 23081697 & 23082323 & 626 & $5.017 \mathrm{E}-15$ & $5.017 \mathrm{E}-15$ & $2 \mathrm{p}-1(1) 1.2 \mathrm{p}+2(0) 1.3 \mathrm{~d}-1(3) 2$ \\
\hline 77 & $2 s^{2} S 2 p^{4}\left({ }^{3} P\right)^{4} P 3 s^{5} P_{1}$ & $1+$ & 23094509 & 23093648 & -861 & $1.684 \mathrm{E}-13$ & $1.684 \mathrm{E}-13$ & $2 \mathrm{~s}+1(1) 1.2 \mathrm{p}+2(0) 1.3 \mathrm{~s}+1(1) 2$ \\
\hline 78 & $2 s^{2} S 2 p^{4}\left({ }^{1} D\right)^{2} D 3 s^{3} D_{2}$ & $2+$ & 23128999 & 23128516 & -484 & $3.537 \mathrm{E}-13$ & $3.537 \mathrm{E}-13$ & $2 \mathrm{~s}+1(1) 1.2 \mathrm{p}+2(4) 3.3 \mathrm{~s}+1(1) 4$ \\
\hline 79 & $2 s^{2} S 2 p^{4}\left({ }^{1} D\right){ }^{2} D 3 s^{3} D_{1}$ & $1+$ & 23157234 & 23156969 & -265 & $5.432 \mathrm{E}-14$ & $5.432 \mathrm{E}-14$ & $2 \mathrm{~s}+1(1) 1.2 \mathrm{p}+2(4) 3.3 \mathrm{~s}+1(1) 2$ \\
\hline 80 & $2 s^{2} S 2 p^{4}\left({ }^{3} P\right){ }^{4} P 3 p^{5} P_{2}^{\circ}$ & $2-$ & 23165854 & 23166004 & 150 & $2.510 \mathrm{E}-13$ & $2.510 \mathrm{E}-13$ & $2 \mathrm{~s}+1(1) 1.2 \mathrm{p}+2(4) 5.3 \mathrm{p}-1(1) 4$ \\
\hline 81 & $2 s^{2} S 2 p^{4}\left({ }^{3} P\right){ }^{4} P 3 p^{5} D_{3}^{\circ}$ & $3-$ & 23186328 & 23186536 & 208 & $1.043 \mathrm{E}-13$ & $1.043 \mathrm{E}-13$ & $2 \mathrm{~s}+1(1) 1.2 \mathrm{p}+2(4) 5.3 \mathrm{p}-1(1) 6$ \\
\hline 82 & $2 s^{2} 2 p^{3}\left({ }^{2} P\right)^{2} P 3 p^{3} D_{3}$ & $3+$ & 23213386 & 23212496 & -891 & $9.651 \mathrm{E}-12$ & $9.651 \mathrm{E}-12$ & $2 p+3(3) 3.3 p+1(3) 6$ \\
\hline 83 & $2 s^{2} S 2 p^{4}\left({ }^{3} P\right)^{4} P 3 s^{3} P_{0}$ & $0+$ & 23218954 & 23218728 & -227 & $8.762 \mathrm{E}-14$ & $8.762 \mathrm{E}-14$ & $2 \mathrm{~s}+1(1) 1.2 \mathrm{p}+2(0) 1.3 \mathrm{~s}+1(1) 0$ \\
\hline 84 & $2 s^{2} 2 p^{3}\left({ }^{2} P\right)^{2} P 3 p^{1} P_{1}$ & $1+$ & 23222776 & 23222019 & -758 & $1.549 \mathrm{E}-12$ & $1.549 \mathrm{E}-12$ & $2 p+3(3) 3.3 p+1(3) 2$ \\
\hline 85 & $2 s^{2} 2 p^{3}\left({ }^{2} P\right)^{2} P 3 p^{3} P_{2}$ & $2+$ & 23274896 & 23274181 & -715 & $1.733 \mathrm{E}-12$ & $1.733 \mathrm{E}-12$ & $2 p+3(3) 3.3 p+1(3) 4$ \\
\hline 86 & $2 s^{2} S 2 p^{4}\left({ }^{3} P\right){ }^{4} P 3 p^{5} D_{4}^{\circ}$ & $4-$ & 23407091 & 23407083 & -8 & $1.195 \mathrm{E}-11$ & $1.195 \mathrm{E}-11$ & $2 \mathrm{~s}+1(1) 1.2 \mathrm{p}+2(4) 5.3 \mathrm{p}+1(3) 8$ \\
\hline 87 & $2 s^{2} S 2 p^{4}\left({ }^{3} P\right){ }^{4} P 3 p^{3} D_{3}^{\circ}$ & $3-$ & 23413650 & 23413685 & 35 & 4.956E-14 & $4.956 \mathrm{E}-14$ & $2 \mathrm{~s}+1(1) 1.2 \mathrm{p}+2(4) 5.3 \mathrm{p}+1(3) 6$ \\
\hline 88 & $2 s^{2} S 2 p^{4}\left({ }^{3} P\right)^{4} P 3 p^{3} P_{1}^{\circ}$ & $1-$ & 23444458 & 23444653 & 195 & 4.504E-14 & $4.504 \mathrm{E}-14$ & $2 \mathrm{~s}+1(1) 1.2 \mathrm{p}+2(4) 5.3 \mathrm{p}+1(3) 2$ \\
\hline 89 & $2 s^{2} 2 p^{3}\left({ }^{2} P\right){ }^{2} P 3 p^{1} S_{0}$ & $0+$ & 23492292 & 23492568 & 276 & $7.453 \mathrm{E}-13$ & $7.453 \mathrm{E}-13$ & $2 p+3(3) 3.3 p+1(3) 0$ \\
\hline 90 & $2 s^{2} S 2 p^{4}\left({ }^{1} S\right){ }^{2} S 3 p^{3} P_{0}^{\circ}$ & $0-$ & 23537382 & 23537683 & 301 & $2.573 \mathrm{E}-13$ & $2.573 \mathrm{E}-13$ & $2 \mathrm{~s}+1(1) 1.2 \mathrm{p}+2(0) 1.3 \mathrm{p}-1(1) 0$ \\
\hline 91 & $2 s^{2} S 2 p^{4}\left({ }^{3} P\right)^{4} P 3 p^{3} D_{2}^{\circ}$ & $2-$ & 23541715 & 23542198 & 483 & $1.184 \mathrm{E}-13$ & $1.184 \mathrm{E}-13$ & $2 \mathrm{~s}+1(1) 1.2 \mathrm{p}+2(4) 5.3 \mathrm{p}+1(3) 4$ \\
\hline 92 & $2 s^{2} S 2 p^{4}\left({ }^{3} P\right){ }^{4} P 3 p^{5} D_{1}^{\circ}$ & $1-$ & 23548591 & 23549121 & 531 & $4.606 \mathrm{E}-14$ & $4.606 \mathrm{E}-14$ & $2 \mathrm{~s}+1(1) 1.2 \mathrm{p}+2(4) 3.3 \mathrm{p}-1(1) 2$ \\
\hline 93 & $2 s^{2} S 2 p^{4}\left({ }^{3} P\right){ }^{4} P 3 p^{3} S_{1}^{0}$ & $1-$ & 23564212 & 23564591 & 379 & $3.439 \mathrm{E}-14$ & $3.439 \mathrm{E}-14$ & $2 \mathrm{~s}+1(1) 1.2 \mathrm{p}+2(0) 1.3 \mathrm{p}-1(1) 2$ \\
\hline 94 & $2 s^{2} S 2 p^{4}\left({ }^{3} P\right)^{4} P 3 p^{3} P_{2}^{\circ}$ & $2-$ & 23577362 & 23578052 & 690 & $2.185 \mathrm{E}-14$ & $2.185 \mathrm{E}-14$ & $2 \mathrm{~s}+1(1) 1.2 \mathrm{p}+2(4) 5.3 \mathrm{p}+1(3) 4$ \\
\hline 95 & $2 s^{2} S 2 p^{4}\left({ }^{3} P\right){ }^{4} P 3 s^{5} P_{2}^{2}$ & $2+$ & 23682878 & 23681954 & -924 & $1.228 \mathrm{E}-13$ & $1.228 \mathrm{E}-13$ & $2 \mathrm{~s}+1(1) 1.2 \mathrm{p}-1(1) 0.2 \mathrm{p}+3(3) 3.3 \mathrm{~s}$ \\
\hline 96 & $2 s^{2} S 2 p^{4}\left({ }^{3} P\right)^{4} P 3 s^{3} P_{1}$ & $1+$ & 261 & 615 & -646 & 14 & -14 & $2 p+3(3) 3$ \\
\hline 97 & $2 s^{2} 2 p^{3}\left({ }^{2} P\right)^{2} P 3 d^{3} P_{0}^{\circ}$ & $0-$ & 23753511 & 23753847 & 337 & $7.562 \mathrm{E}-15$ & $7.562 \mathrm{E}-15$ & $2 p+3(3) 3.3 d-1(3) 0$ \\
\hline 98 & $2 s^{2} S 2 p^{4}\left({ }^{3} P\right)^{2} P 3 p^{1} S_{0}^{\circ}$ & $0-$ & 23779035 & 23779438 & 403 & $2.892 \mathrm{E}-12$ & $2.892 \mathrm{E}-12$ & $2 \mathrm{~s}+1(1) 1.2 \mathrm{p}+2(4) 3.3 \mathrm{p}+1(3) 0$ \\
\hline 99 & $2 s^{2} 2 p^{3}\left({ }^{2} P\right)^{2} P 3 d^{3} P_{1}^{\circ}$ & $1-$ & 23781592 & 23781909 & 316 & $8.751 \mathrm{E}-15$ & $8.751 \mathrm{E}-15$ & $2 p+3(3) 3.3 d-1(3) 2$ \\
\hline 100 & $2 s^{2} 2 p^{3}\left({ }^{2} P\right)^{2} P 3 d^{3} F_{4}^{\circ}$ & $4-$ & 23786871 & 23787263 & 392 & $7.841 \mathrm{E}-11$ & $7.841 \mathrm{E}-11$ & $2 \mathrm{p}+3(3) 3.3 \mathrm{~d}+1(5) 8$ \\
\hline 101 & $2 s^{2} S 2 p^{4}\left({ }^{1} D\right){ }^{2} D 3 p{ }^{4} F_{3}^{\circ}$ & $3-$ & 23787902 & 23788261 & 358 & $4.315 \mathrm{E}-14$ & $4.315 \mathrm{E}-14$ & $2 \mathrm{~s}+1(1) 1.2 \mathrm{p}+2(4) 3.3 \mathrm{p}$ \\
\hline 102 & $2 s^{2} S 2 p^{4}\left({ }^{3} P\right){ }^{4} P 3 p^{5} S_{2}^{0}$ & $2-$ & 23791620 & 23791878 & 258 & $1.948 \mathrm{E}-13$ & $1.948 \mathrm{E}-13$ & $2 p+3(3) 3.3 d+1(5) 4$ \\
\hline 103 & $2 s^{2} 2 p^{3}\left({ }^{2} D\right)^{2} D 3 d^{1} D_{2}^{\circ}$ & $2-$ & 23818232 & 23818527 & 295 & $2.386 \mathrm{E}-13$ & $2.386 \mathrm{E}-13$ & $2 p+3(3) 3.3 d+1(5) 4$ \\
\hline 104 & $2 s^{2} 2 p^{3}\left({ }^{2} P\right)^{2} P 3 d d^{1} F_{3}^{\circ}$ & $3-$ & 23819697 & 23820317 & 620 & $6.830 \mathrm{E}-15$ & $6.830 \mathrm{E}-15$ & $2 p+3(3) 3.3 d-1(3) 6$ \\
\hline 105 & $2 s^{2} S 2 p^{4}\left({ }^{3} P\right)^{4} P 3 p^{3} D_{1}^{\circ}$ & $1-$ & 23837218 & 23837547 & 329 & $2.559 \mathrm{E}-14$ & $2.559 \mathrm{E}-14$ & $2 \mathrm{~s}+1(1) 1.2 \mathrm{p}+2(0) 1.3 \mathrm{p}+1(3) 2$ \\
\hline 106 & $2 s^{2} S 2 p^{4}\left({ }^{3} P\right){ }^{4} P 3 p^{5} D_{2}^{\circ}$ & $2-$ & 23843955 & 23844431 & 476 & E-14 & -14 & $2 p+3(3) 3.3 d-1(3) 4$ \\
\hline 107 & $2 s^{2} 2 p^{3}\left({ }^{4} S\right){ }^{4} S 3 d^{3} D_{2}^{\circ}$ & $2-$ & 23854364 & 54973 & 608 & E-14 & E-14 & $2 p+3(3) 3.3 d-1(3) 4$ \\
\hline 108 & $2 s^{2} 2 p^{3}\left({ }^{2} P\right)^{2} P 3 d^{3} D_{3}^{\circ}$ & $3-$ & 23861693 & 23862126 & 433 & $2.050 \mathrm{E}-13$ & $2.050 \mathrm{E}-13$ & $2 p+3(3) 3.3 d+1(5) 6$ \\
\hline 109 & $2 s^{2} S 2 p^{4}\left({ }^{1} D\right)^{2} D 3 s^{3} D_{3}$ & $3+$ & 23862007 & 23861188 & -820 & $6.398 \mathrm{E}-14$ & $6.398 \mathrm{E}-14$ & $2 \mathrm{~s}+1(1) 1.2 \mathrm{p}-1(1) 2.2 \mathrm{p}+3(3) 5.3$ \\
\hline 110 & $2 s^{2} S 2 p^{4}\left({ }^{3} P\right){ }^{4} P 3 d^{5} D_{3}$ & $3+$ & 23899088 & 23898277 & -811 & $2.026 \mathrm{E}-12$ & $2.026 \mathrm{E}-12$ & $2 \mathrm{~s}+1(1) 1.2 \mathrm{p}+2(4) 5.3 \mathrm{~d}-1(3) 6$ \\
\hline 111 & $2 s^{2} S 2 p^{4}\left({ }^{3} P\right)^{4} P 3 d^{5} D_{2}$ & $2+$ & 23903361 & 23902537 & -824 & $9.447 \mathrm{E}-13$ & $9.447 \mathrm{E}-13$ & $2 \mathrm{~s}+1(1) 1.2 \mathrm{p}+2(4) 5.3 \mathrm{~d}-1(3) 4$ \\
\hline 112 & $2 s^{2} S 2 p^{4}\left({ }^{3} P\right){ }^{4} P 3 d^{5} D_{4}$ & $4+$ & 23916369 & 23915549 & -820 & $1.326 \mathrm{E}-11$ & $1.326 \mathrm{E}-11$ & $2 \mathrm{~s}+1(1) 1.2 \mathrm{p}+2(4) 5.3 \mathrm{~d}-1(3) 8$ \\
\hline 113 & $2 s^{2} S 2 p^{4}\left({ }^{3} P\right){ }^{4} P 3 d^{5} P_{1}$ & $1+$ & 23924709 & 23923905 & -804 & $1.037 \mathrm{E}-13$ & $1.037 \mathrm{E}-13$ & $2 \mathrm{~s}+1(1) 1.2 \mathrm{p}+2(4) 5.3 \mathrm{~d}-1(3) 2$ \\
\hline 114 & $2 s^{2} S 2 p^{4}\left({ }^{1} D\right){ }^{2} D 3 p^{3} D_{1}^{\circ}$ & $1-$ & 23936560 & 23937388 & 828 & $3.786 \mathrm{E}-14$ & $3.786 \mathrm{E}-14$ & $2 \mathrm{~s}+1(1) 1.2 \mathrm{p}+2(4) 3.3 \mathrm{p}+1(3) 2$ \\
\hline 115 & $2 s^{2} S 2 p^{4}\left({ }^{1} D\right)^{2} D 3 s^{1} D_{2}$ & $2+$ & 23941001 & 23940596 & -405 & 7.081E-14 & $1 \mathrm{E}-14$ & $2 \mathrm{~s}+1(1) 1.2 \mathrm{p}-1(1) 2.2 \mathrm{p}+3(3) 5.3$ \\
\hline 116 & $2 s^{2} S 2 p^{4}\left({ }^{3} P\right){ }^{4} P 3 d^{5} F_{5}$ & $5+$ & 23959870 & 23959106 & -764 & $1.837 \mathrm{E}-11$ & $1.837 \mathrm{E}-11$ & $2 s+1(1) 1.2 p+2(4) 5.3 d+1(5) 10$ \\
\hline 117 & $2 s^{2} S 2 p^{4}\left({ }^{3} P\right){ }^{4} P 3 d^{3} F_{4}$ & $4+$ & 24002699 & 24002145 & -554 & $4.271 \mathrm{E}-12$ & $4.271 \mathrm{E}-12$ & $2 \mathrm{~s}+1(1) 1.2 \mathrm{p}+2(4) 5.3 \mathrm{~d}-1(3) 8$ \\
\hline 118 & $2 s^{2} 2 p^{3}\left({ }^{2} P\right)^{2} P 3 d^{1} P_{1}^{\circ}$ & $1-$ & 24007125 & 24007800 & 676 & $3.648 \mathrm{E}-15$ & $3.648 \mathrm{E}-15$ & $2 p+3(3) 3.3 d+1(5) 2$ \\
\hline 119 & $2 s^{2} S 2 p^{4}\left({ }^{3} P\right)^{4} P 3 d^{3} P_{0}$ & $0+$ & 24043050 & 24042440 & -611 & $4.455 \mathrm{E}-14$ & $4.455 \mathrm{E}-14$ & $2 \mathrm{~s}+1(1) 1.2 \mathrm{p}+2(4) 5.3 \mathrm{~d}+1(5) 0$ \\
\hline 120 & $2 s^{2} S 2 p^{4}\left({ }^{3} P\right)^{2} P 3 s^{3} P_{1}$ & $1+$ & 24080223 & 24079741 & -482 & $9.645 \mathrm{E}-14$ & $9.645 \mathrm{E}-14$ & $2 \mathrm{~s}+1(1) 1.2 \mathrm{p}-1(1) 2.2 \mathrm{p}+3(3) 1.3 \mathrm{~s}+1(1) 2$ \\
\hline 121 & $2 s^{2} S 2 p^{4}\left({ }^{3} P\right)^{4} P 3 d^{3} P_{1}$ & $1+$ & 24098456 & 24097870 & -586 & $1.475 \mathrm{E}-14$ & $1.475 \mathrm{E}-14$ & $2 \mathrm{~s}+1(1) 1.2 \mathrm{p}+2(4) 5.3 \mathrm{~d}+1(5) 2$ \\
\hline 122 & $2 s^{2} S 2 p^{4}\left({ }^{3} P\right)^{2} P 3 s^{3} P_{0}$ & $0+$ & 24102121 & 24101864 & -258 & $6.571 \mathrm{E}-14$ & $6.571 \mathrm{E}-14$ & $2 \mathrm{~s}+1(1) 1.2 \mathrm{p}-1(1) 2.2 \mathrm{p}+3(3) 1.3$ \\
\hline 123 & $2 s^{2} S 2 p^{4}\left({ }^{3} P\right)^{4} P 3 p^{5} P_{1}^{\circ}$ & $1-$ & 24114366 & 24114531 & 164 & $6.908 \mathrm{E}-14$ & $6.908 \mathrm{E}-14$ & $2 \mathrm{~s}+1(1) 1.2 \mathrm{p}-1(1) 0.2 \mathrm{p}+3(3) 3.3 \mathrm{p}-1(1) 2$ \\
\hline 124 & $2 s^{2} S 2 p^{4}\left({ }^{3} P\right){ }^{4} P 3 d^{3} F_{3}$ & $3+$ & 24120725 & 24120266 & -459 & $1.295 \mathrm{E}-14$ & $1.295 \mathrm{E}-14$ & $2 \mathrm{~s}+1(1) 1.2 \mathrm{p}+2(4) 5.3 \mathrm{~d}+1(5) 6$ \\
\hline 125 & $2 s^{2} S 2 p^{4}\left({ }^{3} P\right){ }^{4} P 3 d^{3} D_{2}$ & $2+$ & 24142448 & 24141982 & -467 & $9.583 \mathrm{E}-15$ & $9.583 \mathrm{E}-15$ & $2 \mathrm{~s}+1(1) 1.2 \mathrm{p}+2(4) 5.3 \mathrm{~d}+1(5) 4$ \\
\hline 126 & $2 s^{2} S 2 p^{4}\left({ }^{1} D\right){ }^{2} D 3 p^{3} F_{2}^{\circ}$ & $2-$ & 24164804 & 24165094 & 290 & $5.559 \mathrm{E}-14$ & $5.559 \mathrm{E}-14$ & $2 \mathrm{~s}+1(1) 1.2 \mathrm{p}-1(1) 0.2 \mathrm{p}+3(3) 3.3 \mathrm{p}-1(1) 4$ \\
\hline
\end{tabular}


Table 1. (continued)

\begin{tabular}{|c|c|c|c|c|c|c|c|c|}
\hline Key & $S J$-coupled CSF & $J \pi$ & $E_{\mathrm{MCDF}}$ & $E_{\mathrm{MBPT}}$ & $\Delta E$ & MCDF & $\tau_{\mathrm{MBPT}}$ & $j j$-coupled $\mathrm{CSF}^{a, b, c}$ \\
\hline 127 & & $1+$ & & & & & SJL-14 & 年 \\
\hline 128 & $2 p^{4}\left({ }^{3} P\right)$ & $2+$ & 180325 & 180133 & 192 & $00 \mathrm{E}-14$ & 14 & 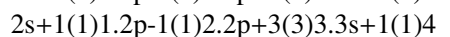 \\
\hline 129 & ${ }^{2} S 2 p^{4}\left({ }^{3} P\right){ }^{2} P 3 d^{3} D_{1}$ & $1+$ & 24278875 & 4278397 & -478 & $.091 \mathrm{E}-13$ & $091 \mathrm{E}-13$ & $2 \mathrm{~s}+1(1) 1.2 \mathrm{p}+2(0) 1.3 \mathrm{~d}-1(3) 2$ \\
\hline 130 & ${ }^{2} S 2 p^{4}\left({ }^{3} P\right){ }^{4} P 3 d^{5} F_{2}$ & $2+$ & 24300148 & 4299642 & -506 & $.128 \mathrm{E}-14$ & $128 \mathrm{E}-14$ & $2 \mathrm{~s}+1(1) 1.2 \mathrm{p}+2(0) 1.3 \mathrm{~d}-1(3) 4$ \\
\hline 131 & $s^{2} S 2 p^{4}\left({ }^{1} D\right){ }^{2} D 3 d^{3} G_{3}$ & $3+$ & 4309230 & 4308888 & -342 & $839 \mathrm{E}-13$ & $839 \mathrm{E}-13$ & $s+1(1) 1.2 p+2(4) 3.3 d-1(3) 6$ \\
\hline 132 & $s^{2} S 2 p^{4}\left({ }^{1} D\right){ }^{2} D 3 p^{3} F_{3}^{\circ}$ & $3-$ & 4313266 & 4313602 & 336 & 7.613E-14 & 7.613E-14 & $2 \mathrm{~s}+1(1) 1.2 \mathrm{p}-1(1) 2.2 \mathrm{p}+3(3) 5.3 \mathrm{p}-1(1) 6$ \\
\hline 133 & $2 s^{2} S 2 p^{4}\left({ }^{3} P\right)^{4} P 3 d^{5} F_{1}{ }^{3}$ & $1+$ & 24324592 & 4324195 & -397 & $6.513 \mathrm{E}-13$ & $6.513 \mathrm{E}-13$ & $2 \mathrm{~s}+1(1) 1.2 \mathrm{p}+2(4) 3.3 \mathrm{~d}-1(3) 2$ \\
\hline 134 & $2 s^{2} S 2 p^{4}\left({ }^{3} P\right){ }^{4} P 3 d^{5} P_{2}$ & $2+$ & 24327534 & 24327238 & -296 & 7.392E-14 & 7.392E-14 & $2 \mathrm{~s}+1(1) 1.2 \mathrm{p}+2(4) 3.3 \mathrm{~d}-1(3) 4$ \\
\hline 135 & $2 s^{2} S 2 p^{4}\left({ }^{1} D\right){ }^{2} D 3 d^{3} P_{0}$ & $0+$ & 4360229 & 1359906 & -323 & $.321 \mathrm{E}-14$ & $321 \mathrm{E}-14$ & $2 \mathrm{~s}+1(1) 1.2 \mathrm{p}+2(4) 3.3 \mathrm{~d}-1(3) 0$ \\
\hline 136 & $2 s^{2} S 2 p^{4}\left({ }^{3} P\right){ }^{4} P 3 p^{5} P_{3}^{\circ}$ & $3-$ & 360230 & 360243 & 13 & $943 \mathrm{E}-14$ & $943 \mathrm{E}-14$ & $2 \mathrm{~s}+1(1) 1.2 \mathrm{p}-1(1) 0.2 \mathrm{p}+3(3) 3$ \\
\hline 137 & $2 s^{2} S 2 p^{4}\left({ }^{1} D\right)^{2} D 3 d^{1} G_{4}$ & $4+$ & 24366985 & 366677 & -308 & $2.316 \mathrm{E}-12$ & $316 \mathrm{E}-12$ & $s+1(1) 1.2 p+2(4) 3.3 d+1(5) 8$ \\
\hline 138 & $2 s^{2} S 2 p^{4}\left({ }^{3} P\right){ }^{4} P 3$ & $3+$ & 24379840 & 24379443 & -397 & 2.08 & $.081 \mathrm{E}-13$ & $s+1(1) 1.2 p+2(4) 3.3 d+1(5) 6$ \\
\hline 139 & $2 s^{2} S 2 p^{4}\left({ }^{1} D\right){ }^{2} D 3 p^{3} P_{1}^{\circ}$ & $1-$ & 24386256 & 24386326 & 70 & $4.343 \mathrm{E}-14$ & 4.343E-14 & $2 \mathrm{~s}+1(1) 1.2 \mathrm{p}-1(1) 0.2 \mathrm{p}+3(3) 3.3 \mathrm{p}+1(3) 2$ \\
\hline 140 & $2 s^{2} S 2 p^{4}\left({ }^{1} D\right){ }^{2} D 3 p^{3} D_{2}^{\circ}$ & $2-$ & 24394336 & 4394538 & 202 & $5.661 \mathrm{E}-14$ & $661 \mathrm{E}-14$ & $2 \mathrm{~s}+1(1) 1.2 \mathrm{p}-1(1) 0.2 \mathrm{p}+3(3) 3.3 \mathrm{p}+1(3) 4$ \\
\hline 141 & $2 s^{2} S 2 p^{4}\left({ }^{3} P\right)^{4} P 3 d^{3} D_{3}{ }^{2}$ & $3+$ & 24416056 & 4415861 & -195 & 8.85 & $8.853 \mathrm{E}-15$ & $2 s+1(1) 1.2 p+2(4)$ \\
\hline 142 & $2 s^{2} S 2 p^{4}\left({ }^{3} P\right){ }^{4} P 3 d^{3} D_{1}$ & $1+$ & 1434711 & 24434324 & -387 & $9.120 \mathrm{E}-15$ & $9.120 \mathrm{E}-15$ & $2 s+1(1)$ \\
\hline 143 & $2 s^{2} S 2 p^{4}\left({ }^{3} P\right){ }^{4} P 3 c$ & $2+$ & 444562 & 4444222 & -340 & 1.6 & -13 & (5) 4 \\
\hline 144 & $2 \mathrm{C}^{2} 441 \mathrm{D}$ & $2-$ & & 59 & 857 & & & 4 \\
\hline 145 & $2 s^{2} S 2 p^{4}\left({ }^{3} P\right)^{2} P 3 p^{3} P_{0}^{\circ}$ & $0-$ & 35 & & 551 & 3.79 & & (1) 0 \\
\hline 146 & $2 s^{2} S 2 p^{4}\left({ }^{3} P\right)^{2} P 3 p^{3} D_{1}^{\circ}$ & $1-$ & 2451 & 64 & 635 & & & \\
\hline 147 & $2 s^{2} S 2 p^{4}\left({ }^{1} D\right){ }^{2} D 3 d^{3} D_{2}$ & $2+$ & 2452 & 95 & 0 & & & \\
\hline 148 & $2 s^{2} S 2 p^{4}\left({ }^{1} D\right){ }^{2} D 3 p^{3} F_{4}^{\circ}$ & $4-$ & 24538583 & 4538751 & 168 & 4.2 & 4.2 & (3) 8 \\
\hline 149 & $2 s^{2} S 2 p^{4}\left({ }^{3} P\right){ }^{4} P 3 p^{3} P_{0}^{\circ}$ & $0-$ & 24545023 & 05 & 682 & & & $+1(3) 0$ \\
\hline 150 & $2 s^{2} S 2 p^{4}\left({ }^{3} P\right)^{2} P 3 p^{1} D_{2}^{\circ}$ & $2-$ & 245 & 530 & 285 & & 14 & (3) 4 \\
\hline 151 & $2 s^{2} S 2 p^{4}\left({ }^{1} D\right)$ & $3-$ & 1593856 & 4594131 & 275 & 5.9 & -14 & $-1(3) 6$ \\
\hline 152 & $2 s^{2} S 2 p^{4}\left({ }^{1} D\right){ }^{2} D 3 p{ }^{1} D_{2}^{\circ}$ & $2-$ & 606863 & 607631 & 767 & 14 & 14 & (1)4 \\
\hline 153 & $2 s^{2} S 2 p^{4}\left({ }^{1} D\right){ }^{2} D 3 p{ }^{1} P_{1}^{\circ}$ & $1-$ & 010 & & 425 & & & (3) 2 \\
\hline 154 & $2 s^{2} S 2 p^{4}\left({ }^{3} P\right)^{2} P 3$ & $1-$ & 223 & 38642 & 1420 & 9.3 & & \\
\hline 155 & $2 s^{2} S 2 p^{4}\left({ }^{3} P\right)^{2} P 3 p^{3} D_{2}^{\circ}$ & $2-$ & 24747317 & 24747707 & 390 & 5.4 & 14 & 1(3)4 \\
\hline 156 & $2 s^{2} S 2 p^{4}\left({ }^{3} P\right)^{2} P 3 p^{3} S_{1}^{2}$ & $1-$ & 24789575 & 24790042 & 467 & & & $-1(3) 2$ \\
\hline 157 & $2 s^{2} S 2 p^{4}\left({ }^{3} P\right){ }^{4} P 3 d^{5} D_{0}$ & $0+$ & 24839947 & 27 & -720 & & & (3) 0 \\
\hline 158 & $2 s^{2} S 2 p^{4}\left({ }^{3} P\right)^{2} P 3 p^{3} D_{3}^{\circ}$ & $3-$ & 06 & 13 & 708 & & & $1(3) 6$ \\
\hline 159 & $2 s^{2} S 2 p^{4}\left({ }^{3} P\right)^{2} P 3 p^{3} P_{2}^{\circ}$ & $2-$ & 45 & 50 & 605 & & & 1(3)4 \\
\hline 160 & $2 s^{2} S 2 p^{4}\left({ }^{3} P\right)$ & $1+$ & & & -730 & & & \\
\hline 161 & $2 s^{2} S 2 p^{4}\left({ }^{1} D\right.$ & $2+$ & & & 0 & & & \\
\hline 162 & ${ }^{4}\left({ }^{3} P\right){ }^{4} P 3$ & $4+$ & & & -( & & & 5) 8 \\
\hline 163 & $2 s^{2} S 2 p^{4}\left({ }^{1} D\right)^{2} D 3 p^{3} P_{0}^{\circ}$ & $0-$ & 786 & 1920725 & 939 & & -12 & $1(3) 0$ \\
\hline 164 & $2 s^{2} S 2 p^{4}\left({ }^{3} P\right){ }^{4} P 3 d^{5} F_{3}$ & $3+$ & 24929143 & 24928562 & -580 & & -14 & $1(3) 6$ \\
\hline 165 & $2 s^{2} S 2 p^{4}\left({ }^{3} P\right){ }^{4} P 3 d^{3} P_{2}$ & $2+$ & 74082 & & -648 & & & $1(5) 4$ \\
\hline 166 & $2 s^{2} S 2 p^{4}\left({ }^{3} P\right)^{2} P 3 p^{1} P_{1}^{\circ}$ & $1-$ & 24975613 & 324 & 1211 & & & $+1(3) 2$ \\
\hline 167 & $2 s^{2} S 2 p^{4}\left({ }^{1} D\right){ }^{2} D 3 d^{3} F_{3}$ & $3+$ & & & -629 & & & 5)6 \\
\hline 168 & $2 s^{2} S 2 p^{4}\left({ }^{1} S\right)$ & $1+$ & & & -860 & & & \\
\hline 169 & $2 s^{2} S 2 p^{4}\left({ }^{1} D\right.$ & $4+$ & & & 5 & & & 8 \\
\hline 170 & $2 s^{2} S 2 p^{4}\left({ }^{1} S\right){ }^{2} S 3 s^{1} S_{0}$ & $0+$ & 97 & & -437 & & e-14 & 0 \\
\hline 171 & $2 s^{2} S 2 p^{4}\left({ }^{1} D\right)^{2} D 3 d^{3} G_{5}$ & $5+$ & 084575 & 84065 & -510 & & E-12 & 5) 10 \\
\hline 172 & $2 s^{2} S 2 p^{4}\left({ }^{1} D\right){ }^{2} D 3 d^{3} S_{1}$ & $1+$ & 091782 & 91097 & -6 & & $\mathrm{E}-15$ & $2 \mathrm{~s}+1(1) 1.2 \mathrm{p}-1(1)$ \\
\hline 173 & $2 s^{2} S 2 p^{4}\left({ }^{1} D\right)^{2} D 3 d^{3} P_{1}$ & $1+$ & 116378 & 856 & -523 & & 5. & $2 s+1(1) 1$ \\
\hline 174 & $2 s^{2} S 2 p^{4}\left({ }^{1} D\right){ }^{2} D 3 d^{3} P_{2}$ & $2+$ & & & -5 & & 15 & 3)4 \\
\hline 175 & $2 s^{2} S 2 p^{4}\left({ }^{1} D\right){ }^{2} D 3 d^{3} D_{3}$ & $3+$ & & & -476 & & & 3) 6 \\
\hline 176 & $2 s^{2} S 2 p^{4}\left({ }^{1} D\right){ }^{2} D 3 d^{3} F_{4}$ & $4+$ & & & -430 & & & \\
\hline 177 & & $3+$ & & & & & & \\
\hline 178 & $2 s^{2} S 2 p^{4}\left({ }^{1} D\right)^{2} D 3 d^{1} D_{2}$ & $2+$ & & & & & & \\
\hline 179 & $2 s^{2} S 2 p^{4}\left({ }^{1} D\right){ }^{2} D 3 d^{1} P_{1}$ & $1+$ & 25237223 & $\delta$ & -3 & & 14 & $2 \mathrm{~s}+1(1) 1.2 \mathrm{p}-1(1) 2.2 \mathrm{p}+$ \\
\hline 180 & $2 s^{2} S 2 p^{4}\left({ }^{1} D\right)^{2} D 3 d^{3} D_{1}$ & $1+$ & 25289570 & 25289238 & -331 & $1.204 \mathrm{E}-14$ & $1.204 \mathrm{E}-14$ & $2 \mathrm{~s}+1(1) 1.2 \mathrm{p}-1(1) 2.2 \mathrm{p}+3(3) 5.3 \mathrm{~d}+1(5) 2$ \\
\hline 181 & $2 s^{2} S 2 p^{4}\left({ }^{3} P\right)^{2} P 3 d^{3} F_{2}$ & $2+$ & 299701 & 299519 & -182 & 14 & E-14 & $2 s+1(1) 1$ \\
\hline 182 & $2 s^{2} S 2 p^{4}\left({ }^{1} D\right){ }^{2} D 3 d^{1} S_{0}$ & $0+$ & 25304627 & 25304509 & -118 & $0 \mathrm{E}-15$ & E-15 & $2 \mathrm{~s}+1(1) 1.2 \mathrm{p}-1(1) 2.2 \mathrm{p}+$ \\
\hline 183 & $2 s^{2} S 2 p^{4}\left({ }^{3} P\right)^{2} P 3 d^{1} F_{3}$ & $3+$ & 25320879 & & -25 & & & $2 \mathrm{~s}+1(1) 1.2 \mathrm{p}-1(1)$ \\
\hline 184 & $2 s^{2} S 2 p^{4}\left({ }^{3} P\right)^{2} P 3 d^{3} F_{3}$ & $3+$ & & & $=$ & & & $d-1(3) 6$ \\
\hline 185 & $2 s^{2} S 2 p^{4}\left({ }^{3} P\right)^{2} P 3 d^{3} P_{2}$ & $2+$ & & 25361604 & -166 & & & $2 \mathrm{~s}+1(1) 1.2 \mathrm{p}-1(1) 2.2 \mathrm{p}+3(3) 1.3 \mathrm{~d}+1(5) 4$ \\
\hline 186 & $2 s^{2} S 2 p^{4}\left({ }^{3} P\right)^{2} P 3 d^{3} D_{2}$ & $2+$ & & & -3 & & & $d-1(3) 4$ \\
\hline 187 & $2 s^{2} S 2 p^{4}\left({ }^{3} P\right)^{2} P 3 d^{3} D_{3}$ & $3+$ & 2745 & 70 & -7 & & 13 & $1(5) 6$ \\
\hline 188 & $2 s^{2} S 2 p^{4}\left({ }^{3} P\right)^{2} P 3 d^{3} F_{4}$ & & 25477 & & 6 & & & 5) 8 \\
\hline 189 & $2 s^{2} S 2 p^{4}\left({ }^{3} P\right){ }^{2} P 3 d^{3} P_{1}$ & $1+$ & 25442114 & 25442087 & -28 & $7.542 \mathrm{E}-15$ & $7.542 \mathrm{E}-15$ & $2 \mathrm{~s}+1(1) 1.2 \mathrm{p}-1(1) 2.2 \mathrm{p}+3(3) 3.3 \mathrm{~d}-1(3) 2$ \\
\hline 190 & $2 s^{2} S 2 p^{4}\left({ }^{3} P\right){ }^{2} P 3 d^{3} P_{0}$ & $0+$ & 25450323 & 25450523 & 19 & $6 \mathrm{E}-15$ & $6.246 \mathrm{E}-15$ & $2 \mathrm{~s}+1(1) 1.2 \mathrm{p}-1(1) 2.2 \mathrm{p}+3(3) 3.3 \mathrm{~d}-1(3) 0$ \\
\hline 191 & $2 s^{2} S 2 p^{4}\left({ }^{3} P\right){ }^{4} P 3 p^{5} D_{0}^{\circ}$ & $0-$ & 25502918 & 25503446 & 528 & $2.076 \mathrm{E}-12$ & $2.076 \mathrm{E}-12$ & $2 \mathrm{~s}+1(1) 1.3 \mathrm{p}-1(1) 0$ \\
\hline
\end{tabular}


Table 1. (continued)

\begin{tabular}{|c|c|c|c|c|c|c|c|c|}
\hline Key & $L S J$-coupled CSF & $J \pi$ & $E_{\mathrm{MCDF}}$ & $E_{\mathrm{MBPT}}$ & $\Delta E$ & $\tau_{\mathrm{MCDF}}$ & $\tau_{\mathrm{MBPT}}$ & $j j$-coupled $\mathrm{CSF}^{a, b, c}$ \\
\hline 192 & $2 s^{2} S 2 p^{4}\left({ }^{3} P\right)^{2} P 3 d^{1} D_{2}$ & $2+$ & 25515508 & 25515873 & 365 & $5.021 \mathrm{E}-15$ & $5.021 \mathrm{E}-15$ & $2 \mathrm{~s}+1(1) 1.2 \mathrm{p}-1(1) 2.2 \mathrm{p}+3(3) 3.3 \mathrm{~d}+1(5) 4$ \\
\hline 193 & $2 s^{2} S 2 p^{4}\left({ }^{3} P\right)^{2} P 3 d^{1} P_{1}$ & $1+$ & 25521091 & 25521081 & -10 & $4.531 \mathrm{E}-15$ & $4.531 \mathrm{E}-15$ & $2 \mathrm{~s}+1(1) 1.2 \mathrm{p}-1(1) 2.2 \mathrm{p}+3(3) 3.3 \mathrm{~d}+1(5) 2$ \\
\hline 194 & $2 s^{2} S 2 p^{4}\left({ }^{1} S\right){ }^{2} S 3 p^{3} P_{1}^{\circ}$ & $1-$ & 25524464 & 25525228 & 764 & $3.352 \mathrm{E}-14$ & $3.352 \mathrm{E}-14$ & $2 \mathrm{~s}+1(1) 1.3 \mathrm{p}-1(1) 2$ \\
\hline 195 & $2 p^{5}\left((5)^{2} P 3 s^{3} P_{2}^{\circ}\right.$ & $2-$ & 25603295 & 25604088 & 793 & $1.042 \mathrm{E}-12$ & $1.042 \mathrm{E}-12$ & $2 p+3(3) 3.3 s+1(1) 4$ \\
\hline 196 & $2 p^{5}\left((5)^{2} P 3 s^{1} P_{1}^{\circ}\right.$ & $1-$ & 25646363 & 25647503 & 1140 & $7.074 \mathrm{E}-14$ & $7.074 \mathrm{E}-14$ & $2 \mathrm{p}+3(3) 3.3 \mathrm{~s}+1(1) 2$ \\
\hline 197 & $2 s^{2} S 2 p^{4}\left({ }^{1} S\right){ }^{2} S 3 p^{3} P_{2}^{\circ}$ & $2-$ & 25691236 & 25691517 & 280 & $4.416 \mathrm{E}-13$ & $4.416 \mathrm{E}-13$ & $2 \mathrm{~s}+1(1) 1.3 \mathrm{p}+1(3) 4$ \\
\hline 198 & $2 s^{2} S 2 p^{4}\left({ }^{1} S\right){ }^{2} S 3 p^{1} P_{1}^{\circ}$ & $1-$ & 25700199 & 25700387 & 189 & $3.201 \mathrm{E}-14$ & $3.201 \mathrm{E}-14$ & $2 \mathrm{~s}+1(1) 1.3 \mathrm{p}+1(3) 2$ \\
\hline 199 & $2 p^{5}\left((5)^{2} P 3 p^{3} S_{1}\right.$ & $1+$ & 26015423 & 26015552 & 129 & $3.997 \mathrm{E}-14$ & $3.997 \mathrm{E}-14$ & $2 p+3(3) 3.3 p-1(1) 2$ \\
\hline 200 & $2 p^{5}\left({ }^{(5)}\right)^{2} P 3 p^{3} D_{2}$ & $2+$ & 26034919 & 26035134 & 214 & $4.112 \mathrm{E}-14$ & $4.112 \mathrm{E}-14$ & $2 \mathrm{p}+3(3) 3.3 \mathrm{p}-1(1) 4$ \\
\hline 201 & $2 s^{2} S 2 p^{4}\left({ }^{1} S\right){ }^{2} S 3 d^{3} D_{3}$ & $3+$ & 26235340 & 26234920 & -420 & $1.138 \mathrm{E}-13$ & $1.138 \mathrm{E}-13$ & $2 \mathrm{~s}+1(1) 1.3 \mathrm{~d}+1(5) 6$ \\
\hline 202 & $2 s^{2} S 2 p^{4}\left({ }^{1} S\right)^{2} S 3 d^{3} D_{1}$ & $1+$ & 26248454 & 26247964 & -490 & $6.585 \mathrm{E}-15$ & $6.585 \mathrm{E}-15$ & $2 \mathrm{~s}+1(1) 1.3 \mathrm{~d}-1(3) 2$ \\
\hline 203 & $2 p^{5}\left((5)^{2} P 3 p^{1} P_{1}\right.$ & $1+$ & 26258920 & 26258969 & 49 & $3.247 \mathrm{E}-14$ & $3.247 \mathrm{E}-14$ & $2 p+3(3) 3.3 p+1(3) 2$ \\
\hline 204 & $2 s^{2} S 2 p^{4}\left({ }^{1} S\right){ }^{2} S 3 d^{3} D_{2}$ & $2+$ & 26265339 & 26265073 & -266 & $8.657 \mathrm{E}-15$ & $8.657 \mathrm{E}-15$ & $2 s+1(1) 1.3 d-1(3) 4$ \\
\hline 205 & $2 p^{5}\left((5)^{2} P 3 p^{3} D_{3}\right.$ & $3+$ & 26265848 & 26265747 & -100 & $5.467 \mathrm{E}-14$ & $5.467 \mathrm{E}-14$ & $2 p+3(3) 3.3 p+1(3) 6$ \\
\hline 206 & $2 p^{5}\left({ }^{(5)}\right)^{2} P 3 p^{3} P_{2}$ & $2+$ & 26297747 & 26297612 & -135 & $2.772 \mathrm{E}-14$ & $2.772 \mathrm{E}-14$ & $2 p+3(3) 3.3 p+1(3) 4$ \\
\hline 207 & $2 s^{2} S 2 p^{4}\left({ }^{1} S\right){ }^{2} S 3 d^{1} D_{2}$ & $2+$ & 26317458 & 26317333 & -125 & $5.496 \mathrm{E}-14$ & $5.496 \mathrm{E}-14$ & $2 p+3(3) 3.3 p+1(3) 4$ \\
\hline 208 & $2 p^{5}\left({ }^{5}\right)^{2} P 3 p^{3} P_{0}$ & $0+$ & 26486871 & 26488001 & 1130 & $3.340 \mathrm{E}-14$ & 3.340E-14 & $2 p+3(3) 3.3 p+1(3) 0$ \\
\hline 209 & $2 p^{5}\left((5)^{2} P 3 s^{3} P_{0}^{\circ}\right.$ & $0-$ & 26510588 & 26511171 & 584 & $9.894 \mathrm{E}-13$ & $9.894 \mathrm{E}-13$ & $2 \mathrm{p}-1(1) 1.3 \mathrm{~s}+1(1) 0$ \\
\hline 210 & $2 p^{5}\left((5)^{2} P 3 s^{3} P_{1}^{\circ}\right.$ & $1-$ & 26533438 & 26534202 & 764 & $8.035 \mathrm{E}-14$ & $8.035 \mathrm{E}-14$ & $2 \mathrm{p}-1(1) 1.3 \mathrm{~s}+1(1) 2$ \\
\hline 211 & $2 p^{5}\left((5)^{2} P 3 d^{3} P_{0}^{\circ}\right.$ & $0-$ & 26716298 & 26717081 & 783 & $7.803 \mathrm{E}-13$ & $7.803 \mathrm{E}-13$ & $2 \mathrm{p}+3(3) 3.3 \mathrm{~d}-1(3) 0$ \\
\hline 212 & $2 p^{5}\left({ }^{5}\right)^{2} P 3 d^{3} P_{1}^{\circ}$ & $1-$ & 26744101 & 26744956 & 854 & $7.735 \mathrm{E}-13$ & $7.735 \mathrm{E}-13$ & $2 \mathrm{p}+3(3) 3.3 \mathrm{~d}-1(3) 2$ \\
\hline 213 & $2 p^{5}\left({ }^{5}\right)^{2} P 3 d^{3} F_{3}^{\circ}$ & $3-$ & 26769316 & 26770513 & 1197 & $7.744 \mathrm{E}-13$ & 7.744E-13 & $2 p+3(3) 3.3 d-1(3) 6$ \\
\hline 214 & $2 p^{5}\left({ }^{(5)}{ }^{2} P 3 d^{3} D_{2}^{\circ}\right.$ & $2-$ & 26791462 & 26792495 & 1034 & $7.673 \mathrm{E}-13$ & $7.673 \mathrm{E}-13$ & $2 \mathrm{p}+3(3) 3.3 \mathrm{~d}-1(3) 4$ \\
\hline 215 & $2 p^{5}\left({ }^{(5)}{ }^{2} P 3 d^{3} F_{4}^{\circ}\right.$ & $4-$ & 26802700 & 26803792 & 1092 & $1.437 \mathrm{E}-12$ & $1.437 \mathrm{E}-12$ & $2 p+3(3) 3.3 d+1(5) 8$ \\
\hline 216 & $2 p^{5}\left({ }^{(5)}{ }^{2} P 3 d^{1} D_{2}^{\circ}\right.$ & $2-$ & 26832698 & 26833736 & 1038 & $1.122 \mathrm{E}-12$ & $1.122 \mathrm{E}-12$ & $2 p+3(3) 3.3 d+1(5) 4$ \\
\hline 217 & $2 p^{5}\left({ }^{(} 5\right)^{2} P 3 d^{3} D_{3}^{\circ}$ & $3-$ & 26872028 & 26873194 & 1167 & $9.005 \mathrm{E}-13$ & $9.005 \mathrm{E}-13$ & $2 p+3(3) 3.3 d+1(5) 6$ \\
\hline 218 & $2 p^{5}(5)^{2} P 3 p^{3} D_{1}^{3}$ & $1+$ & 26926428 & 26926304 & -124 & $3.609 \mathrm{E}-14$ & $3.609 \mathrm{E}-14$ & $2 p-1(1) 1.3 p-1(1) 2$ \\
\hline 219 & $2 p^{5}\left({ }^{(5)}{ }^{2} P 3 d^{1} P_{1}^{\circ}\right.$ & $1-$ & 27001375 & 27002884 & 1509 & $5.012 \mathrm{E}-15$ & $5.012 \mathrm{E}-15$ & $2 p+3(3) 3.3 d+1(5) 2$ \\
\hline 220 & $2 p^{5}(5)^{2} P 3 p^{1} S_{0}$ & $0+$ & 27126800 & 27128336 & 1536 & $2.454 \mathrm{E}-14$ & $2.454 \mathrm{E}-14$ & $2 p-1(1) 1.3 p-1(1) 0$ \\
\hline 221 & $2 p^{5}(5)^{2} P 3 p^{3} P_{1}$ & $1+$ & 27174101 & 27173903 & -199 & $3.414 \mathrm{E}-14$ & $3.414 \mathrm{E}-14$ & $2 \mathrm{p}-1(1) 1.3 \mathrm{p}+1(3) 2$ \\
\hline 222 & $2 p^{5}(5)^{2} P 3 p^{1} D_{2}$ & $2+$ & 27184333 & 27184154 & -178 & $3.657 \mathrm{E}-14$ & $3.657 \mathrm{E}-14$ & $2 \mathrm{p}-1(1) 1.3 \mathrm{p}+1(3) 4$ \\
\hline 223 & $2 p^{5}\left({ }^{5}\right)^{2} P 3 d^{3} F_{2}^{\circ}$ & $2-$ & 27671201 & 27672066 & 865 & $1.098 \mathrm{E}-12$ & $1.098 \mathrm{E}-12$ & $2 \mathrm{p}-1(1) 1.3 \mathrm{~d}-1(3) 4$ \\
\hline 224 & $2 p^{5}\left((5)^{2} P 3 d^{3} P_{2}^{\circ}\right.$ & $2-$ & 27737195 & 27737913 & 718 & $9.126 \mathrm{E}-13$ & $9.126 \mathrm{E}-13$ & $2 \mathrm{p}-1(1) 1.3 \mathrm{~d}+1(5) 4$ \\
\hline 225 & $2 p^{5}\left({ }^{(5)}{ }^{2} P 3 d^{1} F_{3}^{\circ}\right.$ & $3-$ & 27748433 & 27749343 & 910 & $8.899 \mathrm{E}-13$ & $8.899 \mathrm{E}-13$ & $2 \mathrm{p}-1(1) 1.3 \mathrm{~d}+1(5) 6$ \\
\hline 226 & $2 p^{5}\left((5)^{2} P 3 d^{3} D_{1}^{\circ}\right.$ & $1-$ & 27799974 & 27801251 & 1277 & $5.219 \mathrm{E}-15$ & $5.219 \mathrm{E}-15$ & $2 \mathrm{p}-1(1) 1.3 \mathrm{~d}-1(3) 2$ \\
\hline 227 & $2 s^{2} 2 p^{3}\left({ }^{2} D\right)^{2} D 4 s^{3} D_{2}^{\circ}$ & $2-$ & 28153615 & 28154924 & 1309 & $1.604 \mathrm{E}-13$ & $1.604 \mathrm{E}-13$ & $2 \mathrm{p}+1(3) 3.4 \mathrm{~s}+1(1) 4$ \\
\hline 228 & $2 s^{2} 2 p^{3}\left({ }^{2} D\right){ }^{2} D 4 s^{3} D_{1}^{\circ}$ & $1-$ & 28168810 & 28170171 & 1361 & $9.480 \mathrm{E}-14$ & $9.480 \mathrm{E}-14$ & $2 p+1(3) 3.4 s+1(1) 2$ \\
\hline 229 & $2 s^{2} 2 p^{3}\left({ }^{2} D\right)^{2} D 4 p^{3} D_{1}$ & $1+$ & 28336449 & 28336902 & 453 & $1.793 \mathrm{E}-13$ & $1.793 \mathrm{E}-13$ & $2 \mathrm{p}+1(3) 3.4 \mathrm{p}-1(1) 2$ \\
\hline 230 & $2 s^{2} 2 p^{3}\left({ }^{4} S\right){ }^{4} S 4 p^{5} P_{2}$ & $2+$ & 28340331 & 28340699 & 368 & $1.792 \mathrm{E}-13$ & $1.792 \mathrm{E}-13$ & $2 \mathrm{p}+1(3) 3.4 \mathrm{p}-1(1) 4$ \\
\hline 231 & $2 s^{2} 2 p^{3}\left({ }^{2} D\right){ }^{2} D 4 p{ }^{1} F_{3}$ & $3+$ & 28438542 & 28438722 & 180 & $2.046 \mathrm{E}-13$ & $2.046 \mathrm{E}-13$ & $2 p+1(3) 3.4 p+1(3) 6$ \\
\hline 232 & $2 s^{2} 2 p^{3}\left({ }^{2} D\right)^{2} D 4 p^{3} P_{1}$ & $1+$ & 28441026 & 28441362 & 336 & $1.962 \mathrm{E}-13$ & $1.962 \mathrm{E}-13$ & $2 p+1(3) 3.4 p+1(3) 2$ \\
\hline 233 & $2 s^{2} 2 p^{3}\left({ }^{4} S\right){ }^{4} S 4 p^{3} P_{2}$ & $2+$ & 28476873 & 28477132 & 259 & $2.047 \mathrm{E}-13$ & $2.047 \mathrm{E}-13$ & $2 p+1(3) 3.4 p+1(3) 4$ \\
\hline 234 & $2 s^{2} 2 p^{3}\left({ }^{4} S\right){ }^{4} S 4 p^{3} P_{0}$ & $0+$ & 28510339 & 28510933 & 593 & $2.111 \mathrm{E}-13$ & $2.111 \mathrm{E}-13$ & $2 p+1(3) 3.4 p+1(3) 0$ \\
\hline 235 & $2 s^{2} 2 p^{3}\left({ }^{2} P\right)^{2} P 4 d^{3} D_{2}^{\circ}$ & $2-$ & 28629526 & 28630935 & 1409 & $8.362 \mathrm{E}-14$ & $8.362 \mathrm{E}-14$ & $2 \mathrm{p}+1(3) 3.4 \mathrm{~d}-1(3) 4$ \\
\hline 236 & $2 s^{2} 2 p^{3}\left({ }^{2} D\right)^{2} D 4 d^{3} G_{3}^{\circ}$ & $3-$ & 28638159 & 28639415 & 1256 & $9.288 \mathrm{E}-14$ & $9.288 \mathrm{E}-14$ & $2 \mathrm{p}+1(3) 3.4 \mathrm{~d}-1(3) 6$ \\
\hline 237 & $2 s^{2} 2 p^{3}\left({ }^{2} D\right){ }^{2} D 4 d^{3} D_{1}^{\circ}$ & $1-$ & 28643060 & 28644207 & 1147 & $5.376 \mathrm{E}-14$ & $5.376 \mathrm{E}-14$ & $2 p+1(3) 3.4 d-1(3) 2$ \\
\hline 238 & $2 s^{2} 2 p^{3}\left({ }^{2} D\right){ }^{2} D 4 d^{3} P_{0}^{1}$ & $0-$ & 28644326 & 28645366 & 1040 & $9.697 \mathrm{E}-14$ & $9.697 \mathrm{E}-14$ & $2 \mathrm{p}+1(3) 3.4 \mathrm{~d}-1(3) 0$ \\
\hline 239 & $2 s^{2} 2 p^{3}\left({ }^{2} P\right)^{2} P 4 d^{3} F_{4}^{0}$ & $4-$ & 28660718 & 28661851 & 1133 & $9.592 \mathrm{E}-14$ & $9.592 \mathrm{E}-14$ & $2 p+1(3) 3.4 d+1(5) 8$ \\
\hline 240 & $2 s^{2} 2 p^{3}\left({ }^{2} P\right)^{2} P 4 d^{1} D_{2}^{\circ}$ & $2-$ & 28678042 & 28679238 & 1197 & $2.516 \mathrm{E}-14$ & $2.516 \mathrm{E}-14$ & $2 p+1(3) 3.4 d+1(5) 4$ \\
\hline 241 & $2 s^{2} 2 p^{3}\left({ }^{2} P\right)^{2} P 4 d^{1} F_{3}^{\circ}$ & $3-$ & 28706336 & 28707602 & 1265 & $1.272 \mathrm{E}-14$ & $1.272 \mathrm{E}-14$ & $2 p+1(3) 3.4 d+1(5) 6$ \\
\hline 242 & $2 s^{2} 2 p^{3}\left({ }^{2} P\right)^{2} P 4 d^{1} P_{1}^{\circ}$ & $1-$ & 28717020 & 28718330 & 1310 & $1.780 \mathrm{E}-14$ & $1.780 \mathrm{E}-14$ & $2 p+1(3) 3.4 d+1(5) 2$ \\
\hline 243 & $2 s^{2} 2 p^{3}\left({ }^{2} D\right)^{2} D 4 f^{3} G_{3}$ & $3+$ & 28780086 & 28780227 & 141 & $4.518 \mathrm{E}-14$ & $4.518 \mathrm{E}-14$ & $2 p+1(3) 3.4 f-1(5) 6$ \\
\hline 244 & $2 s^{2} 2 p^{3}\left({ }^{2} D\right)^{2} D 4 f^{3} F_{2}$ & $2+$ & 28786709 & 28786770 & 61 & $4.524 \mathrm{E}-14$ & $4.524 \mathrm{E}-14$ & $2 p+1(3) 3.4 f-1(5) 4$ \\
\hline 245 & $2 s^{2} 2 p^{3}\left({ }^{4} S\right){ }^{4} S 4 f^{5} F_{4}$ & $4+$ & 28786803 & 28786933 & 130 & $4.559 \mathrm{E}-14$ & $4.559 \mathrm{E}-14$ & $2 \mathrm{p}+1(3) 3.4 \mathrm{f}-1(5) 8$ \\
\hline 246 & $2 s^{2} 2 p^{3}\left(^{2} P\right)^{2} P 4 f^{3} D_{1}$ & $1+$ & 28795346 & 28795286 & -60 & $4.525 \mathrm{E}-14$ & $4.525 \mathrm{E}-14$ & $2 p+1(3) 3.4 f-1(5) 2$ \\
\hline 247 & $2 s^{2} 2 p^{3}\left({ }^{4} S\right){ }^{4} S 4 f^{3} F_{4}$ & $4+$ & 28796888 & 28797126 & 238 & $4.642 \mathrm{E}-14$ & $4.642 \mathrm{E}-14$ & $2 p+1(3) 3.4 f+1(7) 8$ \\
\hline 248 & $2 s^{2} 2 p^{3}\left({ }^{2} D\right)^{2} D 4 f^{3} D_{3}$ & $3+$ & 28797759 & 28797875 & 116 & $4.566 \mathrm{E}-14$ & $4.566 \mathrm{E}-14$ & $2 p+1(3) 3.4 f+1(7) 6$ \\
\hline 249 & $2 s^{2} 2 p^{3}\left({ }^{2} D\right){ }^{2} D 4 f^{1} H_{5}$ & $5+$ & 28798780 & 28798893 & 113 & $4.597 \mathrm{E}-14$ & $4.597 \mathrm{E}-14$ & $2 p+1(3) 3.4 f+1(7) 10$ \\
\hline 250 & $2 s^{2} 2 p^{3}\left({ }^{2} P\right)^{2} P 4 f^{1} D_{2}$ & $2+$ & 28809948 & 28810014 & 66 & 4.614E-14 & 4.614E-14 & $2 p+1(3) 3.4 f+1(7) 4$ \\
\hline 251 & $2 s^{2} 2 p^{3}\left({ }^{4} S\right){ }^{4} S 4 s^{5} S_{2}^{\circ}$ & $2-$ & 28948743 & 28949863 & 1120 & $1.665 \mathrm{E}-13$ & $1.665 \mathrm{E}-13$ & $2 p-1(1) 1.2 p+2(4) 3.4 s+1(1) 4$ \\
\hline 252 & $2 s^{2} 2 p^{3}\left({ }^{4} S\right){ }^{4} S 4 s^{3} S_{1}^{\circ}$ & $1-$ & 28962444 & 28963605 & 1162 & $9.330 \mathrm{E}-14$ & $9.330 \mathrm{E}-14$ & $2 \mathrm{p}-1(1) 1.2 \mathrm{p}+2(4) 3.4 \mathrm{~s}+1(1) 2$ \\
\hline 253 & $2 s^{2} 2 p^{3}\left({ }^{2} D\right){ }^{2} D 4 s^{3} D_{3}^{\circ}$ & $3-$ & 29077316 & 29078502 & 1187 & $1.656 \mathrm{E}-13$ & $1.656 \mathrm{E}-13$ & $2 \mathrm{p}-1(1) 1.2 \mathrm{p}+2(4) 5.4 \mathrm{~s}+1(1) 6$ \\
\hline 254 & $2 s^{2} 2 p^{3}\left({ }^{2} D\right){ }^{2} D 4 s^{1} D_{2}^{\circ}$ & $2-$ & 29087497 & 29088746 & 1248 & $9.912 \mathrm{E}-14$ & $9.912 \mathrm{E}-14$ & $2 \mathrm{p}-1(1) 1.2 \mathrm{p}+2(4) 5.4 \mathrm{~s}+1(1) 4$ \\
\hline 255 & $2 s^{2} 2 p^{3}\left({ }^{4} S\right){ }^{4} S 4 p^{5} P_{1}$ & $1+$ & 29127260 & 29127537 & 276 & $1.762 \mathrm{E}-13$ & $1.762 \mathrm{E}-13$ & $2 \mathrm{p}-1(1) 1.2 \mathrm{p}+2(4) 3.4 \mathrm{p}-1(1) 2$ \\
\hline 256 & $2 s^{2} 2 p^{3}\left({ }^{2} D\right)^{2} D 4 p^{3} F_{2}$ & $2+$ & 29149379 & 29149613 & 234 & $1.785 \mathrm{E}-13$ & $1.785 \mathrm{E}-13$ & $2 \mathrm{p}-1(1) 1.2 \mathrm{p}+2(4) 3.4 \mathrm{p}-1(1) 4$ \\
\hline
\end{tabular}


Table 1. (continued)

\begin{tabular}{|c|c|c|c|c|c|c|c|c|}
\hline Key & $L S J$-coupled CSF & $J \pi$ & $E_{\mathrm{MCDF}}$ & $E_{\mathrm{MBPT}}$ & $\Delta E$ & $\tau_{\mathrm{MCDF}}$ & $\tau_{\mathrm{MBPT}}$ & $j j$-coupled $\mathrm{CSF}^{a, b, c}$ \\
\hline 257 & $2 s^{2} 2 p^{3}\left({ }^{4} S\right){ }^{4} S 4 p^{5} P_{3}$ & $3+$ & 29231835 & 29231824 & -10 & $2.044 \mathrm{E}-13$ & $2.044 \mathrm{E}-13$ & $2 p-1(1) 1.2 p+2(4) 3.4 p+1(3) 6$ \\
\hline 258 & $2 s^{2} 2 p^{3}\left({ }^{2} D\right)^{2} D 4 p^{3} D_{2}$ & $2+$ & 29236428 & 29236784 & 356 & $2.007 \mathrm{E}-13$ & $2.007 \mathrm{E}-13$ & $2 \mathrm{p}-1(1) 1.2 \mathrm{p}+2(4) 3.4 \mathrm{p}+1(3) 4$ \\
\hline 259 & $2 s^{2} 2 p^{3}\left({ }^{2} D\right){ }^{2} D 4 p^{3} P_{0}$ & $0+$ & 29236687 & 29236879 & 192 & $1.948 \mathrm{E}-13$ & $1.948 \mathrm{E}-13$ & $2 \mathrm{p}-1(1) 1.2 \mathrm{p}+2(4) 3.4 \mathrm{p}+1(3) 0$ \\
\hline 260 & $2 s^{2} 2 p^{3}\left({ }^{2} P\right)^{2} P 4 s^{3} P_{0}^{\circ}$ & $0-$ & 29246189 & 29248331 & 2142 & $1.447 \mathrm{E}-13$ & $1.447 \mathrm{E}-13$ & $2 \mathrm{p}-1(1) 1.2 \mathrm{p}+2(0) 1.4 \mathrm{~s}+1(1) 0$ \\
\hline 261 & $2 s^{2} 2 p^{3}\left({ }^{2} P\right){ }^{2} P 4 s^{3} P_{1}^{\circ}$ & $1-$ & 29251428 & 29253504 & 2076 & $1.204 \mathrm{E}-13$ & $1.204 \mathrm{E}-13$ & $2 \mathrm{p}-1(1) 1.2 \mathrm{p}+2(0) 1.4 \mathrm{~s}+1(1) 2$ \\
\hline 262 & $2 s^{2} 2 p^{3}\left({ }^{2} D\right)^{2} D 4 p^{3} F_{3}$ & $3+$ & 29260375 & 29260486 & 110 & $1.763 \mathrm{E}-13$ & $1.763 \mathrm{E}-13$ & $2 \mathrm{p}-1(1) 1.2 \mathrm{p}+2(4) 5.4 \mathrm{p}-1(1) 6$ \\
\hline 263 & $2 s^{2} 2 p^{3}\left({ }^{4} S\right){ }^{4} S 4 p^{3} P_{1}$ & $1+$ & 29279033 & 29279325 & 293 & $2.033 \mathrm{E}-13$ & $2.033 \mathrm{E}-13$ & $2 \mathrm{p}-1(1) 1.2 \mathrm{p}+2(4) 3.4 \mathrm{p}+1(3) 2$ \\
\hline 264 & $2 s^{2} 2 p^{3}\left({ }^{2} D\right){ }^{2} D 4 p^{3} P_{2}$ & $2+$ & 29293177 & 29293525 & 348 & $1.838 \mathrm{E}-13$ & $1.838 \mathrm{E}-13$ & $2 \mathrm{p}-1(1) 1.2 \mathrm{p}+2(4) 5.4 \mathrm{p}-1(1) 4$ \\
\hline 265 & $2 s^{2} 2 p^{3}\left(^{2} D\right)^{2} D 4 p^{3} D_{3}$ & $3+$ & 29359017 & 29359139 & 121 & $2.000 \mathrm{E}-13$ & $2.000 \mathrm{E}-13$ & $2 \mathrm{p}-1(1) 1.2 \mathrm{p}+2(4) 5.4 \mathrm{p}+1(3) 6$ \\
\hline 266 & $2 s^{2} 2 p^{3}\left({ }^{2} D\right){ }^{2} D 4 p^{3} F_{4}$ & $4+$ & 29361214 & 29361255 & 42 & $2.046 \mathrm{E}-13$ & $2.046 \mathrm{E}-13$ & $2 \mathrm{p}-1(1) 1.2 \mathrm{p}+2(4) 5.4 \mathrm{p}+1(3) 8$ \\
\hline 267 & $2 s^{2} 2 p^{3}\left({ }^{2} D\right){ }^{2} D 4 p^{1} P_{1}$ & $1+$ & 29373041 & 29373924 & 883 & $2.004 \mathrm{E}-13$ & $2.004 \mathrm{E}-13$ & $2 \mathrm{p}-1(1) 1.2 \mathrm{p}+2(4) 5.4 \mathrm{p}+1(3) 2$ \\
\hline 268 & $2 s^{2} 2 p^{3}\left(^{2} D\right)^{2} D 4 p^{1} D_{2}$ & $2+$ & 29418608 & 29418956 & 348 & $2.106 \mathrm{E}-13$ & $2.106 \mathrm{E}-13$ & $2 \mathrm{p}-1(1) 1.2 \mathrm{p}+2(4) 5.4 \mathrm{p}+1(3) 4$ \\
\hline 269 & $2 s^{2} 2 p^{3}\left({ }^{4} S\right){ }^{4} S 4 d^{5} D_{1}^{\circ}$ & $1-$ & 29430093 & 29431197 & 1104 & $6.090 \mathrm{E}-14$ & $6.090 \mathrm{E}-14$ & $2 \mathrm{p}-1(1) 1.2 \mathrm{p}+2(4) 3.4 \mathrm{~d}-1(3) 2$ \\
\hline 270 & $2 s^{2} 2 p^{3}\left({ }^{2} P\right)^{2} P 4 p^{3} D_{1}$ & $1+$ & 29430710 & 29431558 & 847 & $1.780 \mathrm{E}-13$ & $1.780 \mathrm{E}-13$ & $2 \mathrm{p}-1(1) 1.2 \mathrm{p}+2(0) 1.4 \mathrm{p}-1(1) 2$ \\
\hline 271 & $2 s^{2} 2 p^{3}\left({ }^{4} S\right){ }^{4} S 4 d^{5} D_{0}^{\circ}$ & $0-$ & 29434998 & 29436071 & 1072 & $5.723 \mathrm{E}-14$ & $5.723 \mathrm{E}-14$ & $2 \mathrm{p}-1(1) 1.2 \mathrm{p}+2(4) 3.4 \mathrm{~d}-1(3) 0$ \\
\hline 272 & $2 s^{2} 2 p^{3}\left({ }^{4} S\right){ }^{4} S 4 d^{5} D_{2}^{\circ}$ & $2-$ & 29436242 & 29437390 & 1149 & $4.302 \mathrm{E}-14$ & $4.302 \mathrm{E}-14$ & $2 \mathrm{p}-1(1) 1.2 \mathrm{p}+2(4) 3.4 \mathrm{~d}-1(3) 4$ \\
\hline 273 & $2 s^{2} 2 p^{3}\left({ }^{4} S\right){ }^{4} S 4 d^{5} D_{3}^{\circ}$ & $3-$ & 29449321 & 29450457 & 1136 & $3.313 \mathrm{E}-14$ & $3.313 \mathrm{E}-14$ & $2 \mathrm{p}-1(1) 1.2 \mathrm{p}+2(4) 3.4 \mathrm{~d}-1(3) 6$ \\
\hline 274 & $2 s^{2} 2 p^{3}\left({ }^{4} S\right){ }^{4} S 4 d^{5} D_{4}^{\circ}$ & $4-$ & 29453993 & 29454976 & 983 & $9.547 \mathrm{E}-14$ & $9.547 \mathrm{E}-14$ & $2 \mathrm{p}-1(1) 1.2 \mathrm{p}+2(4) 3.4 \mathrm{~d}+1(5) 8$ \\
\hline 275 & $2 s^{2} 2 p^{3}\left({ }^{4} S\right){ }^{4} S 4 d^{3} D_{3}^{\circ}$ & $3-$ & 29460370 & 29461519 & 1148 & $4.980 \mathrm{E}-14$ & $4.980 \mathrm{E}-14$ & $2 \mathrm{p}-1(1) 1.2 \mathrm{p}+2(4) 3.4 \mathrm{~d}+1(5) 6$ \\
\hline 276 & $2 s^{2} 2 p^{3}\left({ }^{2} P\right)^{2} P 4 p^{3} P_{0}^{3}$ & $0+$ & 29482229 & 29483516 & 1286 & $1.863 \mathrm{E}-13$ & $1.863 \mathrm{E}-13$ & $2 \mathrm{p}-1(1) 1.2 \mathrm{p}+2(0) 1.4 \mathrm{p}-1(1) 0$ \\
\hline 277 & $2 s^{2} 2 p^{3}\left({ }^{4} S\right){ }^{4} S 4 d^{3} D_{1}^{\circ}$ & $1-$ & 29490006 & 29491077 & 1070 & $2.294 \mathrm{E}-14$ & $2.294 \mathrm{E}-14$ & $2 \mathrm{p}-1(1) 1.2 \mathrm{p}+2(4) 3.4 \mathrm{~d}+1(5) 2$ \\
\hline 278 & $2 s^{2} 2 p^{3}\left({ }^{4} S\right){ }^{4} S 4 d^{3} D_{2}^{\circ}$ & $2-$ & 29505708 & 29506933 & 1225 & $1.538 \mathrm{E}-14$ & $1.538 \mathrm{E}-14$ & $2 \mathrm{p}-1(1) 1.2 \mathrm{p}+2(4) 3.4 \mathrm{~d}+1(5) 4$ \\
\hline 279 & $2 s^{2} 2 p^{3}\left({ }^{2} P\right)^{2} P 4 p^{3} P_{1}^{2}$ & $1+$ & 29539393 & 29540355 & 961 & $2.040 \mathrm{E}-13$ & $2.040 \mathrm{E}-13$ & $2 \mathrm{p}-1(1) 1.2 \mathrm{p}+2(0) 1.4 \mathrm{p}+1(3) 2$ \\
\hline 280 & $2 s^{2} 2 p^{3}\left({ }^{2} P\right){ }^{2} P 4 p^{3} D_{2}$ & $2+$ & 29544345 & 29545162 & 817 & $2.026 \mathrm{E}-13$ & $2.026 \mathrm{E}-13$ & $2 \mathrm{p}-1(1) 1.2 \mathrm{p}+2(0) 1.4 \mathrm{p}+1(3) 4$ \\
\hline 281 & $2 s^{2} 2 p^{3}\left({ }^{2} D\right){ }^{2} D 4 d^{3} G_{4}^{\circ}$ & $4-$ & 29559189 & 29560168 & 979 & $9.638 \mathrm{E}-14$ & $9.638 \mathrm{E}-14$ & $2 \mathrm{p}-1(1) 1.2 \mathrm{p}+2(4) 5.4 \mathrm{~d}-1(3) 8$ \\
\hline 282 & $2 s^{2} 2 p^{3}\left({ }^{2} D\right)^{2} D 4 d^{3} D_{3}^{\circ}$ & $3-$ & 29571520 & 29572646 & 1126 & $2.633 \mathrm{E}-14$ & $2.633 \mathrm{E}-14$ & $2 \mathrm{p}-1(1) 1.2 \mathrm{p}+2(4) 5.4 \mathrm{~d}-1(3) 6$ \\
\hline 283 & $2 s^{2} 2 p^{3}\left({ }^{2} D\right)^{2} D 4 d^{3} F_{4}^{\circ}$ & $4-$ & 29580866 & 29581848 & 982 & $9.532 \mathrm{E}-14$ & $9.532 \mathrm{E}-14$ & $2 \mathrm{p}-1(1) 1.2 \mathrm{p}+2(4) 5.4 \mathrm{~d}+1(5) 8$ \\
\hline 284 & $2 s^{2} 2 p^{3}\left({ }^{4} S\right){ }^{4} S 4 f^{5} F_{3}$ & $3+$ & 29581384 & 29581408 & 24 & $4.531 \mathrm{E}-14$ & $4.531 \mathrm{E}-14$ & $2 \mathrm{p}-1(1) 1.2 \mathrm{p}+2(4) 3.4 \mathrm{f}-1(5) 6$ \\
\hline 285 & $2 s^{2} 2 p^{3}\left({ }^{4} S\right){ }^{4} S 4 f^{5} F_{2}$ & $2+$ & 29582534 & 29582488 & -46 & $4.493 \mathrm{E}-14$ & $4.493 \mathrm{E}-14$ & $2 \mathrm{p}-1(1) 1.2 \mathrm{p}+2(4) 3.4 \mathrm{f}-1(5) 4$ \\
\hline 286 & $2 s^{2} 2 p^{3}\left({ }^{2} D\right)^{2} D 4 d^{3} D_{2}^{\circ}$ & $2-$ & 29583203 & 29584426 & 1223 & $1.759 \mathrm{E}-14$ & $1.759 \mathrm{E}-14$ & $2 \mathrm{p}-1(1) 1.2 \mathrm{p}+2(4) 5.4 \mathrm{~d}-1(3) 4$ \\
\hline 287 & $2 s^{2} 2 p^{3}\left({ }^{2} D\right)^{2} D 4 f^{3} H_{4}$ & $4+$ & 29583489 & 29583560 & 71 & $4.579 \mathrm{E}-14$ & $4.579 \mathrm{E}-14$ & $2 \mathrm{p}-1(1) 1.2 \mathrm{p}+2(4) 3.4 \mathrm{f}-1(5) 8$ \\
\hline 288 & $2 s^{2} 2 p^{3}\left({ }^{2} D\right){ }^{2} D 4 d^{3} G_{5}^{\circ}$ & $5-$ & 29584086 & 29585038 & 952 & $9.558 \mathrm{E}-14$ & $9.558 \mathrm{E}-14$ & $2 \mathrm{p}-1(1) 1.2 \mathrm{p}+2(4) 5.4 \mathrm{~d}+1(5) 10$ \\
\hline 289 & $2 s^{2} 2 p^{3}\left({ }^{4} S\right){ }^{4} S 4 f^{5} F_{1}$ & $1+$ & 29586615 & 29586524 & -91 & $4.489 \mathrm{E}-14$ & $4.489 \mathrm{E}-14$ & $2 \mathrm{p}-1(1) 1.2 \mathrm{p}+2(4) 3.4 \mathrm{f}-1(5) 2$ \\
\hline 290 & $2 s^{2} 2 p^{3}\left({ }^{2} D\right)^{2} D 4 d^{3} P_{1}^{\circ}$ & $1-$ & 29591177 & 29592433 & 1256 & $1.725 \mathrm{E}-14$ & $1.725 \mathrm{E}-14$ & $2 \mathrm{p}-1(1) 1.2 \mathrm{p}+2(4) 5.4 \mathrm{~d}-1(3) 2$ \\
\hline 291 & $2 s^{2} 2 p^{3}\left({ }^{2} D\right){ }^{2} D 4 f^{1} G_{4}$ & $4+$ & 29591930 & 29591957 & 26 & $4.556 \mathrm{E}-14$ & $4.556 \mathrm{E}-14$ & $2 \mathrm{p}-1(1) 1.2 \mathrm{p}+2(4) 3.4 \mathrm{f}+1(7) 8$ \\
\hline 292 & $2 s^{2} 2 p^{3}\left({ }^{4} S\right){ }^{4} S 4 f^{5} F_{5}$ & $5+$ & 29592653 & 29592677 & 24 & $4.574 \mathrm{E}-14$ & $4.574 \mathrm{E}-14$ & $2 \mathrm{p}-1(1) 1.2 \mathrm{p}+2(4) 3.4 \mathrm{f}+1(7) 10$ \\
\hline 293 & $2 s^{2} 2 p^{3}\left({ }^{2} D\right)^{2} D 4 d^{1} S_{0}^{\circ}$ & $0-$ & 29594969 & 29596178 & 1209 & 3.003E-14 & $3.003 \mathrm{E}-14$ & $2 \mathrm{p}-1(1) 1.2 \mathrm{p}+2(4) 5.4 \mathrm{~d}+1(5) 0$ \\
\hline 294 & $2 s^{2} 2 p^{3}\left({ }^{4} S\right){ }^{4} S 4 f^{3} F_{3}$ & $3+$ & 29597550 & 29597606 & 55 & $4.586 \mathrm{E}-14$ & $4.586 \mathrm{E}-14$ & $2 p-1(1) 1.2 p+2(4) 3.4 f+1(7) 6$ \\
\hline 295 & $2 s^{2} 2 p^{3}\left({ }^{4} S\right){ }^{4} S 4 f^{3} F_{2}$ & $2+$ & 29600693 & 29600687 & -6 & $4.569 \mathrm{E}-14$ & $4.569 \mathrm{E}-14$ & $2 \mathrm{p}-1(1) 1.2 \mathrm{p}+2(4) 3.4 \mathrm{f}+1(7) 4$ \\
\hline 296 & $2 s^{2} 2 p^{3}\left({ }^{2} D\right)^{2} D 4 d^{3} S_{1}^{\circ}$ & $1-$ & 29617671 & 29618902 & 1230 & $1.601 \mathrm{E}-14$ & $1.601 \mathrm{E}-14$ & $2 \mathrm{p}-1(1) 1.2 \mathrm{p}+2(4) 5.4 \mathrm{~d}+1(5) 2$ \\
\hline 297 & $2 s^{2} 2 p^{3}\left({ }^{2} D\right){ }^{2} D 4 d^{1} D_{2}^{\circ}$ & $2-$ & 29629837 & 29631117 & 1280 & $1.557 \mathrm{E}-14$ & $1.557 \mathrm{E}-14$ & $2 \mathrm{p}-1(1) 1.2 \mathrm{p}+2(4) 5.4 \mathrm{~d}+1(5) 4$ \\
\hline 298 & $2 s^{2} 2 p^{3}\left({ }^{2} D\right){ }^{2} D 4 d^{1} F_{3}^{\circ}$ & $3-$ & 29630987 & 29632140 & 1152 & $1.504 \mathrm{E}-14$ & $1.504 \mathrm{E}-14$ & $2 \mathrm{p}-1(1) 1.2 \mathrm{p}+2(4) 5.4 \mathrm{~d}+1(5) 6$ \\
\hline 299 & $2 s^{2} 2 p^{3}\left({ }^{2} D\right){ }^{2} D 4 f^{3} H_{5}$ & $5+$ & 29707410 & 29707484 & 74 & $4.578 \mathrm{E}-14$ & $4.578 \mathrm{E}-14$ & $2 \mathrm{p}-1(1) 1.2 \mathrm{p}+2(4) 5.4 \mathrm{f}-1(5) 10$ \\
\hline 300 & $2 s^{2} 2 p^{3}\left({ }^{2} D\right){ }^{2} D 4 f^{3} G_{4}$ & $4+$ & 29710182 & 29710158 & -25 & $4.539 \mathrm{E}-14$ & $4.539 \mathrm{E}-14$ & $2 \mathrm{p}-1(1) 1.2 \mathrm{p}+2(4) 5.4 \mathrm{f}-1(5) 8$ \\
\hline 301 & $2 s^{2} 2 p^{3}\left({ }^{2} D\right){ }^{2} D 4 f^{3} F_{3}$ & $3+$ & 29711323 & 29711234 & -89 & $4.505 \mathrm{E}-14$ & $4.505 \mathrm{E}-14$ & $2 p-1(1) 1.2 p+2(4) 5.4 f-1(5) 6$ \\
\hline 302 & $2 s^{2} 2 p^{3}\left({ }^{2} D\right)^{2} D 4 f^{3} D_{2}$ & $2+$ & 29713264 & 29713141 & -124 & $4.483 \mathrm{E}-14$ & $4.483 \mathrm{E}-14$ & $2 \mathrm{p}-1(1) 1.2 \mathrm{p}+2(4) 5.4 \mathrm{f}-1(5) 4$ \\
\hline 303 & $2 s^{2} 2 p^{3}\left({ }^{2} D\right){ }^{2} D 4 f^{3} D_{1}$ & $1+$ & 29715228 & 29715083 & -145 & $4.476 \mathrm{E}-14$ & $4.476 \mathrm{E}-14$ & $2 \mathrm{p}-1(1) 1.2 \mathrm{p}+2(4) 5.4 \mathrm{f}-1(5) 2$ \\
\hline 304 & $2 s^{2} 2 p^{3}\left({ }^{2} D\right){ }^{2} D 4 f^{3} P_{0}$ & $0+$ & 29716529 & 29716377 & -152 & $4.480 \mathrm{E}-14$ & $4.480 \mathrm{E}-14$ & $2 \mathrm{p}-1(1) 1.2 \mathrm{p}+2(4) 5.4 \mathrm{f}-1(5) 0$ \\
\hline 305 & $2 s^{2} 2 p^{3}\left({ }^{2} D\right){ }^{2} D 4 f^{3} H_{6}$ & $6+$ & 29718692 & 29718763 & 71 & 4.603E-14 & 4.603E-14 & $2 \mathrm{p}-1(1) 1.2 \mathrm{p}+2(4) 5.4 \mathrm{f}+1(7) 12$ \\
\hline 306 & $2 s^{2} 2 p^{3}\left({ }^{2} D\right)^{2} D 4 f^{3} G_{5}$ & $5+$ & 29719620 & 29719596 & -23 & $4.557 \mathrm{E}-14$ & $4.557 \mathrm{E}-14$ & $2 \mathrm{p}-1(1) 1.2 \mathrm{p}+2(4) 5.4 \mathrm{f}+1(7) 10$ \\
\hline 307 & $2 s^{2} 2 p^{3}\left({ }^{2} D\right)^{2} D 4 f^{3} F_{4}$ & $4+$ & 29723846 & 29723835 & -11 & $4.578 \mathrm{E}-14$ & $4.578 \mathrm{E}-14$ & $2 \mathrm{p}-1(1) 1.2 \mathrm{p}+2(4) 5.4 \mathrm{f}+1(7) 8$ \\
\hline 308 & $2 s^{2} 2 p^{3}\left({ }^{2} D\right)^{2} D 4 f^{1} P_{1}$ & $1+$ & 29725929 & 29725774 & -155 & 4.489E-14 & $4.489 \mathrm{E}-14$ & $2 \mathrm{p}-1(1) 1.2 \mathrm{p}+2(4) 5.4 \mathrm{f}+1(7) 2$ \\
\hline 309 & $2 s^{2} 2 p^{3}\left({ }^{2} D\right){ }^{2} D 4 f^{1} F_{3}$ & $3+$ & 29726441 & 29726399 & -41 & $4.562 \mathrm{E}-14$ & $4.562 \mathrm{E}-14$ & $2 \mathrm{p}-1(1) 1.2 \mathrm{p}+2(4) 5.4 \mathrm{f}+1(7) 6$ \\
\hline 310 & $2 s^{2} 2 p^{3}\left({ }^{2} D\right)^{2} D 4 f^{1} D_{2}$ & $2+$ & 29726863 & 29726771 & -92 & $4.526 \mathrm{E}-14$ & $4.526 \mathrm{E}-14$ & $2 \mathrm{p}-1(1) 1.2 \mathrm{p}+2(4) 5.4 \mathrm{f}+1(7) 4$ \\
\hline 311 & $2 s^{2} 2 p^{3}\left({ }^{2} P\right)^{2} P 4 d^{3} F_{2}^{\circ}$ & $2-$ & 29731200 & 29732936 & 1736 & 5.399E-14 & $5.399 \mathrm{E}-14$ & $2 \mathrm{p}-1(1) 1.2 \mathrm{p}+2(0) 1.4 \mathrm{~d}-1(3) 4$ \\
\hline 312 & $2 s^{2} 2 p^{3}\left({ }^{2} P\right)^{2} P 4 d^{3} P_{2}^{\circ}$ & $2-$ & 29765678 & 29767493 & 1815 & $2.792 \mathrm{E}-14$ & $2.792 \mathrm{E}-14$ & $2 \mathrm{p}-1(1) 1.2 \mathrm{p}+2(0) 1.4 \mathrm{~d}+1(5) 4$ \\
\hline 313 & $2 s^{2} 2 p^{3}\left({ }^{2} P\right)^{2} P 4 d^{3} D_{1}^{\circ}$ & $1-$ & 29768117 & 29770185 & 2068 & $1.492 \mathrm{E}-14$ & $1.492 \mathrm{E}-14$ & $2 \mathrm{p}-1(1) 1.2 \mathrm{p}+2(0) 1.4 \mathrm{~d}-1(3) 2$ \\
\hline 314 & $2 s^{2} 2 p^{3}\left({ }^{2} P\right)^{2} P 4 d^{3} F_{3}^{\circ}$ & $3-$ & 29771761 & 29773468 & 1707 & $2.829 \mathrm{E}-14$ & $2.829 \mathrm{E}-14$ & $2 \mathrm{p}-1(1) 1.2 \mathrm{p}+2(0) 1.4 \mathrm{~d}+1(5) 6$ \\
\hline 315 & $2 s^{2} 2 p^{3}\left({ }^{2} P\right)^{2} P 4 f^{3} G_{3}$ & $3+$ & 29878769 & 29879390 & 620 & 4.609E-14 & $4.609 \mathrm{E}-14$ & $2 \mathrm{p}-1(1) 1.2 \mathrm{p}+2(0) 1.4 \mathrm{f}-1(5) 6$ \\
\hline 316 & $2 s^{2} 2 p^{3}\left({ }^{2} P\right)^{2} P 4 f^{3} F_{2}$ & $2+$ & 29882953 & 29883528 & 575 & $4.610 \mathrm{E}-14$ & $4.610 \mathrm{E}-14$ & $2 p-1(1) 1.2 p+2(0) 1.4 f-1(5) 4$ \\
\hline 317 & $2 s^{2} 2 p^{3}\left({ }^{2} P\right)^{2} P 4 f^{3} G_{4}$ & $4+$ & 29891679 & 29892359 & 680 & $4.660 \mathrm{E}-14$ & $4.660 \mathrm{E}-14$ & $2 \mathrm{p}-1(1) 1.2 \mathrm{p}+2(0) 1.4 \mathrm{f}+1(7) 8$ \\
\hline 318 & $2 s^{2} 2 p^{3}\left({ }^{2} P\right)^{2} P 4 f^{3} D_{3}$ & $3+$ & 29892128 & 29892669 & 541 & $4.609 \mathrm{E}-14$ & $4.609 \mathrm{E}-14$ & $2 p-1(1) 1.2 p+2(0) 1.4 f+1(7) 6$ \\
\hline
\end{tabular}

${ }^{a}$ The number at the end or in parentheses is $2 J$.

$b_{s+}=s_{1 / 2}, p-=p_{1 / 2}, p+=p_{3 / 2}, d-=d_{3 / 2}, d+=d_{5 / 2}, f-=f_{5 / 2}$, and $f+=f_{7 / 2}$. 
${ }^{c}$ The number after \pm is the occupation number of the corresponding sub-shell. For example, the $j j$-coupled CSF of level 9 is $2 s_{1 / 2} 2 p_{1 / 2} 2 p_{3 / 2}^{4}$. The full sub-shell $2 p_{3 / 2}$ is omitted. 
Table 2. Wavelengths $\left(\lambda\right.$, in vacuum $\AA$ ), transition rates $\left(A\right.$, in $\left.\mathrm{s}^{-1}\right)$, oscillator strengths $(g f$, dimensionless $)$, line strengths $(S$, in a.u.), and branching fractions (BF) for the transitions among the 318 levels listed in Table 1 from the present MBPT and MCDF calculations. The two first columns ( $i$ and $j$ ) refer to the keys of Table 1 The last column represents the estimated accuracies of the $S$ values using the terminology of the NIST ASD [46]. Only transitions with BF $\geq 10^{-5}$ are presented.

\begin{tabular}{|c|c|c|c|c|c|c|c|c|c|c|c|c|c|}
\hline$i$ & $j$ & Type & $\lambda_{\mathrm{MBPT}}$ & $A_{\mathrm{MBPT}}$ & $g f_{\mathrm{MBPT}}$ & $S_{\mathrm{MBPT}}$ & $\mathrm{BF}_{\mathrm{MBPT}}$ & $\lambda_{\mathrm{MCDF}}$ & $A_{\mathrm{MCDF}}$ & $g f_{\mathrm{MCDF}}$ & $S_{\mathrm{MCDF}}$ & $\mathrm{BF}_{\mathrm{MCDF}}$ & Acc. \\
\hline 1 & 2 & E2 & $4.81296 \mathrm{E}+02$ & $1.252 \mathrm{E}+01$ & $4.348 \mathrm{E}-10$ & $2.887 \mathrm{E}-04$ & $1.000 \mathrm{E}+00$ & $4.81163 \mathrm{E}+02$ & $1.243 \mathrm{E}+01$ & $4.314 \mathrm{E}-10$ & $2.862 \mathrm{E}-04$ & $1.000 \mathrm{E}+00$ & $\mathrm{~B}+$ \\
\hline 1 & 3 & M1 & $1.15783 \mathrm{E}+02$ & $1.066 \mathrm{E}+07$ & $6.430 \mathrm{E}-05$ & $1.841 \mathrm{E}+00$ & $8.279 \mathrm{E}-01$ & $1.15846 \mathrm{E}+02$ & $1.066 \mathrm{E}+07$ & $6.435 \mathrm{E}-05$ & $1.844 \mathrm{E}+00$ & $8.280 \mathrm{E}-01$ & AA \\
\hline 1 & 3 & E2 & $1.15783 \mathrm{E}+02$ & $4.675 E+03$ & $2.819 \mathrm{E}-08$ & $2.605 \mathrm{E}-04$ & $3.631 \mathrm{E}-04$ & $1.15846 \mathrm{E}+02$ & $4.610 \mathrm{E}+03$ & $2.782 \mathrm{E}-08$ & $2.576 \mathrm{E}-04$ & $3.581 \mathrm{E}-04$ & $\mathrm{~B}+$ \\
\hline 2 & 3 & M1 & $1.52459 \mathrm{E}+02$ & $2.211 \mathrm{E}+06$ & $2.311 \mathrm{E}-05$ & $8.713 \mathrm{E}-01$ & $1.717 \mathrm{E}-01$ & $1.52582 \mathrm{E}+02$ & $2.209 \mathrm{E}+06$ & $2.313 \mathrm{E}-05$ & $8.728 \mathrm{E}-01$ & $1.716 \mathrm{E}-01$ & AA \\
\hline 1 & 4 & M1 & $1.02378 \mathrm{E}+02$ & $7.057 \mathrm{E}+06$ & $5.545 \mathrm{E}-05$ & $1.404 \mathrm{E}+00$ & $9.979 \mathrm{E}-01$ & $1.02414 \mathrm{E}+02$ & $7.056 \mathrm{E}+06$ & $5.548 \mathrm{E}-05$ & $1.405 \mathrm{E}+00$ & $9.977 \mathrm{E}-01$ & $\mathrm{AA}$ \\
\hline 1 & 4 & E2 & $1.02378 \mathrm{E}+02$ & $9.136 \mathrm{E}+03$ & $7.180 \mathrm{E}-08$ & 4.587E-04 & $1.292 \mathrm{E}-03$ & $1.02414 \mathrm{E}+02$ & $9.036 \mathrm{E}+03$ & 7.105E-08 & $4.545 \mathrm{E}-04$ & $1.278 \mathrm{E}-03$ & $\mathrm{~B}+$ \\
\hline 2 & 4 & E2 & $1.30040 \mathrm{E}+02$ & $8.323 \mathrm{E}+02$ & $1.055 \mathrm{E}-08$ & $1.382 \mathrm{E}-04$ & $1.177 \mathrm{E}-04$ & $1.30106 \mathrm{E}+02$ & $8.266 \mathrm{E}+02$ & $1.049 \mathrm{E}-08$ & $1.376 \mathrm{E}-04$ & $1.169 \mathrm{E}-04$ & $\mathrm{~B}+$ \\
\hline 3 & 4 & M1 & $8.84311 \mathrm{E}+02$ & $5.022 \mathrm{E}+03$ & $2.944 \mathrm{E}-06$ & $6.438 \mathrm{E}-01$ & $7.101 \mathrm{E}-04$ & $8.83264 \mathrm{E}+02$ & $5.043 \mathrm{E}+03$ & $2.949 \mathrm{E}-06$ & $6.442 \mathrm{E}-01$ & 7.131E-04 & $\mathrm{AA}$ \\
\hline 3 & 5 & M1 & $9.45353 \mathrm{E}+01$ & $3.516 \mathrm{E}+07$ & $4.710 \mathrm{E}-05$ & $1.101 \mathrm{E}+00$ & $9.992 \mathrm{E}-01$ & $9.45670 \mathrm{E}+01$ & $3.517 \mathrm{E}+07$ & $4.715 \mathrm{E}-05$ & $1.103 \mathrm{E}+00$ & $9.992 \mathrm{E}-01$ & $\mathrm{AA}$ \\
\hline 4 & 5 & E2 & $1.05851 \mathrm{E}+02$ & $2.954 \mathrm{E}+04$ & $4.962 \mathrm{E}-08$ & $3.505 \mathrm{E}-04$ & $8.394 \mathrm{E}-04$ & $1.05906 \mathrm{E}+02$ & $2.923 \mathrm{E}+04$ & $4.915 \mathrm{E}-08$ & $3.477 \mathrm{E}-04$ & $8.304 \mathrm{E}-04$ & $\mathrm{~B}+$ \\
\hline 1 & 6 & E1 & $4.20220 \mathrm{E}+01$ & $1.720 \mathrm{E}+11$ & $2.277 \mathrm{E}-01$ & $3.150 \mathrm{E}-02$ & $8.493 \mathrm{E}-01$ & $4.20105 \mathrm{E}+01$ & $1.677 \mathrm{E}+11$ & $2.219 \mathrm{E}-01$ & $3.069 \mathrm{E}-02$ & $8.491 \mathrm{E}-01$ & $\mathrm{~B}+$ \\
\hline 3 & 6 & E1 & $6.59623 \mathrm{E}+01$ & $1.905 \mathrm{E}+10$ & $6.213 \mathrm{E}-02$ & $1.349 \mathrm{E}-02$ & $9.407 \mathrm{E}-02$ & $6.59135 \mathrm{E}+01$ & $1.861 \mathrm{E}+10$ & $6.062 \mathrm{E}-02$ & $1.315 \mathrm{E}-02$ & $9.422 \mathrm{E}-02$ & $\mathrm{~B}+$ \\
\hline 4 & 6 & E1 & $7.12791 \mathrm{E}+01$ & $1.146 \mathrm{E}+10$ & $4.365 \mathrm{E}-02$ & $1.024 \mathrm{E}-02$ & $5.659 \mathrm{E}-02$ & $7.12289 \mathrm{E}+01$ & $1.119 \mathrm{E}+10$ & $4.256 \mathrm{E}-02$ & $9.981 \mathrm{E}-03$ & $5.665 \mathrm{E}-02$ & $\mathrm{~B}+$ \\
\hline 1 & 7 & E1 & $3.76767 \mathrm{E}+01$ & $3.046 \mathrm{E}+11$ & $1.945 \mathrm{E}-01$ & $2.412 \mathrm{E}-02$ & 6.987E-01 & $3.76635 \mathrm{E}+01$ & $2.972 \mathrm{E}+11$ & $1.896 \mathrm{E}-01$ & $2.351 \mathrm{E}-02$ & $6.978 \mathrm{E}-01$ & $\mathrm{~B}+$ \\
\hline 2 & 7 & E1 & $4.08766 \mathrm{E}+01$ & $9.258 \mathrm{E}+10$ & $6.957 \mathrm{E}-02$ & $9.362 \mathrm{E}-03$ & $2.124 \mathrm{E}-01$ & $4.08620 \mathrm{E}+01$ & $9.073 \mathrm{E}+10$ & $6.814 \mathrm{E}-02$ & $9.166 \mathrm{E}-03$ & $2.130 \mathrm{E}-01$ & $\mathrm{~B}+$ \\
\hline 3 & 7 & E1 & $5.58512 \mathrm{E}+01$ & $1.985 \mathrm{E}+10$ & $2.785 \mathrm{E}-02$ & $5.120 \mathrm{E}-03$ & $4.553 \mathrm{E}-02$ & $5.58074 \mathrm{E}+01$ & $1.940 \mathrm{E}+10$ & $2.717 \mathrm{E}-02$ & $4.993 \mathrm{E}-03$ & $4.555 \mathrm{E}-02$ & $\mathrm{~B}+$ \\
\hline 4 & 7 & E1 & $5.96164 \mathrm{E}+01$ & $1.868 \mathrm{E}+10$ & $2.986 \mathrm{E}-02$ & $5.861 \mathrm{E}-03$ & $4.285 \mathrm{E}-02$ & $5.95714 \mathrm{E}+01$ & $1.828 \mathrm{E}+10$ & $2.918 \mathrm{E}-02$ & $5.722 \mathrm{E}-03$ & $4.292 \mathrm{E}-02$ & $\mathrm{~B}+$ \\
\hline 5 & 7 & E1 & $1.36488 \mathrm{E}+02$ & $2.250 \mathrm{E}+08$ & $1.885 \mathrm{E}-03$ & $8.470 \mathrm{E}-04$ & $5.161 \mathrm{E}-04$ & $1.36161 \mathrm{E}+02$ & $2.178 \mathrm{E}+08$ & $1.816 \mathrm{E}-03$ & $8.142 \mathrm{E}-04$ & $5.114 \mathrm{E}-04$ & B \\
\hline 3 & 8 & E1 & $4.12216 \mathrm{E}+01$ & $3.267 \mathrm{E}+11$ & $8.322 \mathrm{E}-02$ & $1.129 \mathrm{E}-02$ & $1.000 \mathrm{E}+00$ & $4.12097 \mathrm{E}+01$ & $3.183 \mathrm{E}+11$ & $8.104 \mathrm{E}-02$ & $1.099 \mathrm{E}-02$ & $9.998 \mathrm{E}-01$ & $\mathrm{~B}+$ \\
\hline 7 & 8 & M1 & $1.57370 \mathrm{E}+02$ & $8.553 \mathrm{E}+06$ & $3.177 \mathrm{E}-05$ & $1.236 \mathrm{E}+00$ & $2.618 \mathrm{E}-05$ & $1.57545 \mathrm{E}+02$ & $8.545 \mathrm{E}+06$ & $3.180 \mathrm{E}-05$ & $1.239 \mathrm{E}+00$ & $2.684 \mathrm{E}-05$ & AA \\
\hline 1 & 9 & E1 & $2.83309 \mathrm{E}+01$ & $3.138 \mathrm{E}+10$ & $1.133 \mathrm{E}-02$ & $1.057 \mathrm{E}-03$ & $5.789 \mathrm{E}-02$ & $2.83251 \mathrm{E}+01$ & $3.091 \mathrm{E}+10$ & $1.115 \mathrm{E}-02$ & $1.040 \mathrm{E}-03$ & $5.830 \mathrm{E}-02$ & $\mathrm{~B}+$ \\
\hline 2 & 9 & E1 & $3.01028 \mathrm{E}+01$ & $2.491 \mathrm{E}+09$ & $1.015 \mathrm{E}-03$ & $1.006 \mathrm{E}-04$ & $4.595 \mathrm{E}-03$ & $3.00968 \mathrm{E}+01$ & $2.605 \mathrm{E}+09$ & $1.061 \mathrm{E}-03$ & $1.052 \mathrm{E}-04$ & $4.913 \mathrm{E}-03$ & B \\
\hline 3 & 9 & E1 & $3.75089 \mathrm{E}+01$ & $4.121 \mathrm{E}+10$ & $2.608 \mathrm{E}-02$ & $3.220 \mathrm{E}-03$ & $7.602 \mathrm{E}-02$ & $3.74921 \mathrm{E}+01$ & $4.009 \mathrm{E}+10$ & $2.535 \mathrm{E}-02$ & $3.129 \mathrm{E}-03$ & $7.561 \mathrm{E}-02$ & $\mathrm{~B}+$ \\
\hline 4 & 9 & E1 & $3.91703 \mathrm{E}+01$ & $4.447 \mathrm{E}+11$ & $3.069 \mathrm{E}-01$ & 3.957E-02 & $8.204 \mathrm{E}-01$ & $3.91541 \mathrm{E}+01$ & $4.346 \mathrm{E}+11$ & $2.997 \mathrm{E}-01$ & $3.863 \mathrm{E}-02$ & $8.197 \mathrm{E}-01$ & $\mathrm{~B}+$ \\
\hline 5 & 9 & E1 & $6.21802 \mathrm{E}+01$ & $2.227 \mathrm{E}+10$ & $3.872 \mathrm{E}-02$ & $7.927 \mathrm{E}-03$ & 4.108E-02 & $6.21205 \mathrm{E}+01$ & $2.190 \mathrm{E}+10$ & $3.801 \mathrm{E}-02$ & $7.773 \mathrm{E}-03$ & $4.130 \mathrm{E}-02$ & $\mathrm{~B}+$ \\
\hline 6 & 9 & M1 & $8.69553 \mathrm{E}+01$ & $1.268 \mathrm{E}+07$ & $4.311 \mathrm{E}-05$ & $9.270 \mathrm{E}-01$ & $2.339 \mathrm{E}-05$ & $8.69500 \mathrm{E}+01$ & $1.268 \mathrm{E}+07$ & 4.313E-05 & $9.274 \mathrm{E}-01$ & $2.391 \mathrm{E}-05$ & AA \\
\hline 7 & 10 & E1 & $3.82035 \mathrm{E}+01$ & $5.076 \mathrm{E}+11$ & $1.111 \mathrm{E}-01$ & $1.397 \mathrm{E}-02$ & $6.704 \mathrm{E}-01$ & $3.82074 \mathrm{E}+01$ & $4.917 \mathrm{E}+11$ & $1.076 \mathrm{E}-01$ & $1.354 \mathrm{E}-02$ & $6.697 \mathrm{E}-01$ & $\mathrm{~B}+$ \\
\hline 9 & 10 & E1 & $5.74054 \mathrm{E}+01$ & $2.496 \mathrm{E}+11$ & $1.233 \mathrm{E}-01$ & $2.330 \mathrm{E}-02$ & $3.296 \mathrm{E}-01$ & $5.74071 \mathrm{E}+01$ & $2.425 \mathrm{E}+11$ & $1.198 \mathrm{E}-01$ & $2.264 \mathrm{E}-02$ & 3.303E-01 & $\mathrm{B}+$ \\
\hline
\end{tabular}

Only transitions among the lowest 10 levels of the $n=2$ configurations are shown here. Table 2 is available online in its entirety at the JQSRT website. 
Table 3. Energy levels (in $\mathrm{cm}^{-1}$ ) relative to the ground state for the $n=2$ states. NIST - the experimental values from the NIST ASD [46]; MBPT - the present MBPT results; MCDF - the present MCDF results; Gu - the theoretical values from Gu [7]; Vilkas - the theoretical values from Vilkas et al. [9]. $\Delta E$ - the differences (in $\mathrm{cm}^{-1}$ ) between calculated energies from different calculations and the NIST values.

\begin{tabular}{|c|c|c|c|c|c|c|c|c|c|c|}
\hline \multirow[t]{2}{*}{ Key } & \multirow[t]{2}{*}{ State } & \multirow{2}{*}{$\begin{array}{c}\text { NIST } \\
E\end{array}$} & \multicolumn{2}{|c|}{ MBPT } & \multicolumn{2}{|c|}{ MCDF } & \multicolumn{2}{|c|}{$\mathrm{Gu}$} & \multicolumn{2}{|c|}{ Vilkas } \\
\hline & & & E & $\Delta E$ & E & $\Delta E$ & E & $\Delta E$ & E & $\Delta E$ \\
\hline 1 & $2 s^{2} 2 p^{4}\left({ }^{3} P\right){ }^{3} P_{2}$ & 0 & 0 & & 0 & & 0 & & 0 & \\
\hline 2 & $2 s^{2} 2 p^{4}\left({ }^{1} S\right){ }^{1} S_{0}$ & 208080 & 207772 & -308 & 207830 & -250 & 207878 & -202 & 207956 & -124 \\
\hline 3 & $2 s^{2} 2 p^{4}\left({ }^{3} P\right){ }^{3} P_{1}$ & 863620 & 863686 & 66 & 863215 & -405 & 864158 & 538 & 864147 & 527 \\
\hline 4 & $2 s^{2} 2 p^{4}\left({ }^{1} D\right){ }^{1} D_{2}$ & 976560 & 976768 & 208 & 976432 & -128 & 977254 & 694 & 977113 & 553 \\
\hline 5 & $2 s^{2} 2 p^{4}\left({ }^{3} P\right){ }^{3} P_{0}$ & 1920420 & 1921492 & 1072 & 1920667 & 247 & 1922438 & 2018 & 1922041 & 1621 \\
\hline 6 & $2 s^{2} S 2 p^{5}{ }^{3} P_{2}^{\circ}$ & 2380360 & 2379704 & -656 & 2380355 & -5 & 2381134 & 774 & 2382023 & 1663 \\
\hline 7 & $2 s^{2} S 2 p^{5}{ }^{3} P_{1}^{\circ}$ & 2654560 & 2654158 & -402 & 2655091 & 531 & 2655814 & 1254 & 2656837 & 2277 \\
\hline 8 & $2 s^{2} S 2 p^{5}{ }^{3} P_{0}^{\circ}$ & 3289690 & 3289601 & -89 & 3289830 & 140 & 3291540 & 1850 & 3292303 & 2613 \\
\hline 9 & $2 s^{2} S 2 p^{5}{ }^{1} P_{1}^{\circ}$ & 3530130 & 3529720 & -410 & 3530442 & 312 & 3531756 & 1626 & 3532737 & 2607 \\
\hline 10 & $2 p^{6}{ }^{1} S_{0}$ & 5273440 & 5271717 & -1723 & 5272387 & -1053 & 5274365 & 925 & 5279298 & 5858 \\
\hline
\end{tabular}


Table 4. Comparison of wavelengths $\left(\lambda\right.$ in $\AA$ ) and transition rates $\left(A\right.$ in $\left.s^{-1}\right)$ for some transitions among the $\mathrm{n}=2$ states of O-like Mo. NIST - the experimental wavelengths from the NIST ASD [46]; MBPT - the present MBPT results; MCDF - the present MCDF results.

\begin{tabular}{|c|c|c|c|c|c|c|c|c|c|c|c|}
\hline \multirow[t]{2}{*}{$i$} & \multirow[t]{2}{*}{$j$} & \multirow[t]{2}{*}{ Lower level } & \multirow[t]{2}{*}{ Upper level } & \multicolumn{3}{|c|}{$\lambda$} & \multicolumn{2}{|c|}{ Percentage difference for $\lambda$} & \multicolumn{2}{|c|}{$A$} & \multirow{2}{*}{$\frac{\text { Percentage difference for } A}{\text { MBPT\& MCDF }}$} \\
\hline & & & & NIST & MBPT & MCDF & NIST \& MBPT & NIST\& MCDF & MBPT & $\mathrm{MCDF}$ & \\
\hline 3 & 9 & $2 s^{2} 2 p^{4}\left({ }^{3} P\right){ }^{3} P_{1}$ & $2 s^{2} S 2 p^{5}{ }^{1} P_{1}^{\circ}$ & 37.483 & 37.5089 & 37.4921 & 0.07 & 0.02 & $4.121 \mathrm{E}+10$ & $4.009 \mathrm{E}+10$ & -3 \\
\hline 1 & 7 & $2 s^{2} 2 p^{4}\left({ }^{3} P\right){ }^{3} P_{2}$ & $2 s^{2} S 2 p^{5}{ }^{3} P_{1}^{\circ}$ & 37.661 & 37.6767 & 37.6635 & 0.04 & 0.01 & $3.046 \mathrm{E}+11$ & $2.972 \mathrm{E}+11$ & -2 \\
\hline 7 & 10 & $2 s^{2} S 2 p^{53} P_{1}^{\circ}$ & $2 p^{6}{ }^{1} S_{0}$ & 38.187 & 38.2035 & 38.2074 & 0.04 & 0.05 & $5.076 \mathrm{E}+11$ & $4.917 \mathrm{E}+11$ & -3 \\
\hline 4 & 9 & $2 s^{2} 2 p^{4}\left({ }^{1} D\right){ }^{1} D_{2}$ & $2 s^{2} S 2 p^{5}{ }^{1} P_{1}^{\circ}$ & 39.161 & 39.1703 & 39.1541 & 0.02 & -0.02 & $4.447 \mathrm{E}+11$ & $4.346 \mathrm{E}+11$ & -2 \\
\hline 2 & 7 & $2 s^{2} 2 p^{4}\left({ }^{1} S\right){ }^{1} S_{0}$ & $2 s^{2} S 2 p^{5}{ }^{3} P_{1}^{\circ}$ & 40.866 & 40.8766 & 40.8620 & 0.03 & -0.01 & $9.258 \mathrm{E}+10$ & $9.073 \mathrm{E}+10$ & -2 \\
\hline 3 & 8 & $2 s^{2} 2 p^{4}\left({ }^{3} P\right){ }^{3} P_{1}$ & $2 s^{2} S 2 p^{5}{ }^{3} P_{0}^{\circ}$ & 41.221 & 41.2216 & 41.2097 & 0.00 & -0.03 & $3.267 \mathrm{E}+11$ & $3.183 \mathrm{E}+11$ & -3 \\
\hline 1 & 6 & $2 s^{2} 2 p^{4}\left({ }^{3} P\right)^{3} P_{2}$ & $2 s^{2} S 2 p^{53} P_{2}^{\circ}$ & 42.014 & 42.0220 & 42.0105 & 0.02 & -0.01 & $1.720 \mathrm{E}+11$ & $1.677 \mathrm{E}+11$ & -3 \\
\hline 3 & 7 & $2 s^{2} 2 p^{4}\left({ }^{3} P\right){ }^{3} P_{1}$ & $2 s^{2} S 2 p^{53} P_{1}^{\circ}$ & 55.842 & 55.8512 & 55.8074 & 0.02 & -0.06 & $1.985 \mathrm{E}+10$ & $1.940 \mathrm{E}+10$ & -2 \\
\hline 9 & 10 & $2 s^{2} S 2 p^{5}{ }^{1} P_{1}^{\circ}$ & $2 p^{6}{ }^{1} S_{0}$ & 57.362 & 57.4054 & 57.4071 & 0.08 & 0.08 & $2.496 \mathrm{E}+11$ & $2.425 \mathrm{E}+11$ & -3 \\
\hline 4 & 7 & $2 s^{2} 2 p^{4}\left({ }^{1} D\right){ }^{1} D_{2}$ & $2 s^{2} S 2 p^{53} P_{1}^{\circ}$ & 59.590 & 59.6164 & 59.5714 & 0.04 & -0.03 & $1.868 \mathrm{E}+10$ & $1.828 \mathrm{E}+10$ & -2 \\
\hline 5 & 9 & $2 s^{2} 2 p^{4}\left({ }^{3} P\right)^{3} P_{0}$ & $2 s^{2} S 2 p^{5}{ }^{1} P_{1}^{\circ}$ & 62.135 & 62.1802 & 62.1205 & 0.07 & -0.02 & $2.227 \mathrm{E}+10$ & $2.190 \mathrm{E}+10$ & -2 \\
\hline 3 & 6 & $2 s^{2} 2 p^{4}\left({ }^{3} P\right){ }^{3} P_{1}$ & $2 s^{2} S 2 p^{53} P_{2}^{\circ}$ & 65.933 & 65.9623 & 65.9135 & 0.04 & -0.03 & $1.905 \mathrm{E}+10$ & $1.861 \mathrm{E}+10$ & -2 \\
\hline 4 & 6 & $2 s^{2} 2 p^{4}\left({ }^{1} D\right){ }^{1} D_{2}$ & $2 s^{2} S 2 p^{53} P_{2}^{\circ}$ & 71.223 & 71.2791 & 71.2289 & 0.08 & 0.01 & $1.146 \mathrm{E}+10$ & $1.119 \mathrm{E}+10$ & -2 \\
\hline
\end{tabular}

